\title{
Branched ubiquitin chain binding and deubiquitination by UCH37 facilitate proteasome clearance of stress-induced inclusions
}

\author{
Aixin Song ${ }^{1}$, Zachary Hazlett ${ }^{1}$, Dulith Abeykoon ${ }^{2}$, Jeremy Dortch ${ }^{1}$, Andrew Dillon ${ }^{1}$, Justin Curtiss ${ }^{1}$, \\ Sarah Bollinger Martinez ${ }^{1}$, Christopher P. Hill ${ }^{3}$, Clinton $\mathrm{Yu}^{4}$, Lan Huang ${ }^{4}$, David Fushman ${ }^{3}$, Robert \\ E. Cohen ${ }^{1}$ and Tingting Yao ${ }^{1 *}$
}

1. Department of Biochemistry and Molecular Biology, Colorado State University, Fort Collins, CO 80523

2. Department of Chemistry and Biochemistry, University of Maryland, College Park, MD 20742

3. Department of Biochemistry, University of Utah School of Medicine, Salt Lake City, UT 84112

4. Department of Physics and Biophysics, University of California, Irvine, CA 92697

*Corresponding author: tingting.yao@colostate.edu 


\section{Abstract}

UCH37, also known as UCHL5, is a highly conserved deubiquitinating enzyme (DUB) that associates with the $26 \mathrm{~S}$ proteasome. Recently it was reported that $\mathrm{UCH} 37$ activity is stimulated by branched ubiquitin chain architectures. To understand how UCH37 achieves its unique debranching specificity, we performed biochemical and NMR structural analyses and found that $\mathrm{UCH} 37$ is activated by contacts with the hydrophobic patches of both distal ubiquitins that emanate from a branched ubiquitin. In addition, RPN13, which recruits UCH37 to the proteasome, further enhances branched-chain specificity by restricting linear ubiquitin chains from having access to the UCH37 active site. In cultured human cells under conditions of proteolytic stress, we show that substrate clearance by the proteasome is promoted by both binding and deubiquitination of branched polyubiquitin by $\mathrm{UCH} 37$. Proteasomes containing $\mathrm{UCH} 37(\mathrm{C} 88 \mathrm{~A})$, which is catalytically inactive, aberrantly retain polyubiquitinated species as well as the RAD23B substrate shuttle factor, suggesting a defect in recycling of the proteasome. These findings provide a foundation to understand how proteasome degradation of substrates modified by a unique ubiquitin chain architecture is aided by a DUB. 


\section{Introduction}

In eukaryotes, the ubiquitin (Ub)-proteasome system is responsible for regulated protein degradation that maintains protein homeostasis and plays a major role in the defense against proteolytic stresses. Ub in the form of a variety of polyUb chains serves as a signal that targets unwanted proteins to the $26 \mathrm{~S}$ proteasome where the proteins are unfolded and translocated into the interior chamber of the 20S proteasome where they are degraded. During this process, $\mathrm{Ub}$ is removed and recycled by deubiquitinating enzymes (DUBs). The timing of Ub removal is critical as premature deubiquitination can lead to unintended release of the substrate, whereas failure to remove Ub can inhibit substrate translocation. Additionally, polyUb chains released from the substrates need to be further disassembled in order to minimize competition with new substrates awaiting processing by the proteasome. Coordination of these actions relies on three DUBs associated with the proteasome: RPN11, USP14 and UCH37. Each of these DUBs has a non-redundant role in proteasome function. In recent years, significant progress has been made in our understanding of how RPN11 and USP14 are regulated to coordinate with substrate processing on the $26 \mathrm{~S}$ proteasome, but the role of $\mathrm{UCH} 37$ remains poorly understood.

Proteasome-associated DUBs can either promote or inhibit substrate degradation ${ }^{1}$. RPN11 (also called POH1) is a stoichiometric subunit of the 19S Regulatory Particle (RP) of the proteasome; it is the only essential DUB in Saccharomyces cerevisiae. RPN11 activity is coupled to substrate degradation by removing polyUb chains en bloc as the substrate is translocated through a narrow gate into the proteolytic chamber of the 20 s proteasome ${ }^{2-4}$. USP14 (UBP6 in yeast) is a dissociable subunit of the $19 \mathrm{~S} \mathrm{RP}^{5}$. It is activated upon forming a complex with the RP and removes Ub from substrates that are ubiquitinated at multiple sites ${ }^{6}$. In vivo and in vitro evidence suggest that USP14/UBP6 inhibits proteasome degradation via both catalytic and noncatalytic mechanisms ${ }^{6-9}$. In contrast to RPN11, USP14 can act before substrate is translocated into the $20 \mathrm{~S}$ core particle and committed to degradation. Inhibition of proteasome-associated DUBs have broad effects on cellular proteostasis ${ }^{10,11}$, highlighting the therapeutic potential of targeting proteasomal DUBs. 
UCH37 (also known as UCHL5) is a highly conserved DUB found from fission yeast to man, but absent in the budding yeast S. cerevisiae. It has a catalytic domain characteristic of the UCH family of DUBs and a unique C-terminal domain (CTD) that mediates interactions with two binding partners, RPN13 $3^{12-14}$ and NFRKB. We previously reported that RPN13 (also known as ADRM1) recruits $\mathrm{UCH} 37$ to the $19 \mathrm{~S} \mathrm{RP}$ of the proteasome whereas NFRKB recruits it to the INO80 chromatin remodeler ${ }^{15}$. Remarkably, whereas UCH37 is activated upon binding RPN13, it is held inactive in the complex with INO80. We and others have solved the crystal structures of $\mathrm{UCH} 37$ in both the activated and repressed states ${ }^{16,17}$. These structures revealed that, whereas RPN13 stabilizes UCH37 in a conformation that promotes Ub binding, NFRKB represses UCH37 by occluding the Ub binding site. Despite that these structures provided clear understanding of how $\mathrm{UCH} 37$ activity is controlled in different contexts, how UCH37 contributes to the functions of the proteasome or INO80 complex remains unknown. Deletion of UCH37 in mice is embryonic lethal, although the underlying cause is unclear ${ }^{18}$. Cellular functions that have been associated with UCH37 include TGF $\beta$ signaling ${ }^{19}$, Hedgehog signaling ${ }^{20}$, DNA repair ${ }^{21}$, cell cycle regulation ${ }^{22}$; in these cases and others ${ }^{23,24} \mathrm{UCH} 37$ was reported to either promote or inhibit degradation of a proposed substrate. However, whether the effects seen on specific substrates were direct or indirect were not resolved, and therefore $\mathrm{UCH} 37$ 's role in proteasome-mediated degradation remains enigmatic.

Among the variety of polyUb polymers in the cell, $10-20 \%$ have a branched architecture ${ }^{25}$ in which more than one Ub is attached to a single Ub acceptor. Branched polyUb chains have been reported to have a variety of functions ${ }^{26}$; most notably, polyUb branching enhances substrate degradation by the proteasome 27,28 . Recently, Deol et al. revealed that UCH37 has a K48 linkage-specific debranching activity that promotes proteasomal degradation of substrates modified with branched polyUb ${ }^{29}$. Here, we set out to understand how the debranching specificity is achieved and how such an activity might contribute to proteasome function in vivo. Our work demonstrates that $\mathrm{UCH} 37$ binds to branched polyUb by engaging the hydrophobic patches on both distal ubiquitins at a branch point. RPN13 further enhances the 
branched chain specificity by restricting UCH37 from engaging linear polyUb chains. In cells, polyUb conjugates that accumulate upon proteolytic stresses are greatly enhanced by the loss of UCH37 activity. We show that both binding and deubiquitination of branched polyUb by UCH37 facilitate proteasome-dependent clearance of stress-induced inclusions.

\section{Results}

\section{UCH37 and the UCH37-RPN13 ${ }^{\mathrm{C}}$ complex prefer branched polyUb substrates}

It was recently reported that UCH37 DUB activity with polyUb substrates is stimulated by branched-chain architectures ${ }^{29}$. To better understand this unusual specificity, we generated homotypic and heterotypic $\mathrm{Ub}_{3}$ chains containing all-native isopeptide linkages and having linear or branched architectures (Figure S1A). We also compared deubiquitination with UCH37 alone or in a complex with the minimal binding domain from RPN13 (RPN13C; also known as the DEUBAD domain ${ }^{30}$ )(Figure $1 A$ and $1 B$ ). By quantifying the release of the $U b_{2}$ and $U b_{1}$ products (Figure 1C), we drew the following conclusions: (1) The branched $\mathrm{Ub}_{3}$ chains are strongly preferred as substrates (i.e., 10 to 100 -fold faster hydrolysis) over their linear counterparts (e.g., [Ub] $2_{2^{-6,48}} \mathrm{Ub}$ versus Ub- ${ }^{6} \mathrm{Ub}_{-}{ }^{48} \mathrm{Ub}$ or $\mathrm{Ub}^{48} \mathrm{Ub}-{ }^{6} \mathrm{Ub}$ ). (2) Among the branched $\mathrm{Ub}_{3}$ substrates, UCH37 strongly prefers K6/K48 over K11/K48 or K48/K63 branched chains. (3) Whereas RPN13 ${ }^{\mathrm{C}}$ enhances UCH37 hydrolysis of most polyUb substrates, it strongly inhibits activity with linear K48 $\mathrm{Ub}_{3}$ (Figure $1 \mathrm{C}$ ). (4) Consistently, we found that $\mathrm{Ub}_{2}$ and $\mathrm{Ub}_{1}$ products were always produced in a 2:1 ratio (Figure $1 \mathrm{C}$ ). Using linkage-specific anti-Ub antibodies, we found that only the K48 linkage in K6/K48 branched Ub 3 was cleaved (Figure S1B), indicative of a "debranching" activity that thus far is unique to $\mathrm{UCH} 37$. This observation is consistent with a previous report that UCH37 exclusively cleaves the K48 linkages in branched polyUb ${ }^{29}$.

By promoting hydrolysis of branched polyUb chains while suppressing disassembly of linear K48 Ub chains, RPN13 ${ }^{\mathrm{C}}$ potentiates the debranching specificity of $\mathrm{UCH} 37$. In the crystal structures of UCH37 in complex with Ub and RPN13 ${ }^{C}$, we and others have observed that RPN13 ${ }^{C}$ interacts with two distinct regions of $\mathrm{UCH} 37^{16,17}$ : in addition to binding the $\mathrm{UCH} 37^{\mathrm{CTD}}, \mathrm{RPN} 13^{\mathrm{C}}$ also makes 
contacts with UCH37 active site crossover loop (ASCL) residues M148 and F149. These latter contacts restrict the conformation of the ASCL and promote UbAMC hydrolysis ${ }^{16,17}$ (Figure S1C). Mutations of ASCL residues M148 and F149 to alanines (AA) or aspartates (DD) had little effects on hydrolysis of the branched $[\mathrm{Ub}]_{2^{-}}{ }^{6,48} \mathrm{Ub}$ substrate, but they abolished the ability of RPN13 ${ }^{\mathrm{C}}$ to suppress linear $\mathrm{K} 48 \mathrm{Ub}_{3}$ hydrolysis (Figure $1 \mathrm{D}$ and $1 \mathrm{E}$ ). Thus, RPN13 ${ }^{\mathrm{C}}$ contacts with the UCH37 ASCL play an unexpected role to enhance UCH37 substrate specificity for branched polyUb.

\section{UCH37-RPN13 ${ }^{\mathrm{C}}$ preferentially binds and deubiquitinates $\mathrm{K} 6 / \mathrm{K} 48$ branched $\mathrm{Ub}_{3}$}

To better understand the factors contributing to UCH37 substrate specificity, we measured the binding affinities of catalytically-inactive $\mathrm{UCH} 37(\mathrm{C} 88 \mathrm{~S})-\mathrm{RPN} 13^{\mathrm{C}}$ with linear and branched Ub chains using microscale thermophoresis (Figure $2 \mathrm{~A}$ and S2A). UCH37(C88S)-RPN13 ${ }^{\mathrm{C}}$ showed highest affinity for $[\mathrm{Ub}]_{2^{-}}{ }^{6,48} \mathrm{Ub}\left(K_{\mathrm{D}}=4.9 \mu \mathrm{M}\right)$ whereas its affinities for [Ub] $2^{-11,48} \mathrm{Ub}$ and $[\mathrm{Ub}]_{2^{-}}$ 48,63 Ub were substantially weaker and similar to those for linear Ub3. Thus, substrate binding only partially accounts for UCH37 specificity.

In order to determine the kinetics of debranching, we took advantage of a previously developed free Ub sensor, $\mathrm{tUI}$, which specifically and tightly $\left(K_{\mathrm{D}}=10^{-10} \mathrm{M}\right)$ binds to mono or polyUb that has a free C-terminus (G76) ${ }^{31}$. Upon cleavage of the $\mathrm{K} 48$ linkage in a branched $\mathrm{Ub}_{3}$, free $\mathrm{Ub}$ is released and captured quantitatively by Atto532-labeled tUI, leading to a fluorescence increase that can be monitored in real time (Figure 2B). To prevent binding of the substrate by Atto532tUI, the proximal Ub in each substrate was modified by deletion of the C-terminal diglycine $(\mathrm{Ub} \Delta \mathrm{GG})$. Using this free Ub sensor-based assay, we determined the $K_{\mathrm{M}}$ and $k_{\text {cat }}$ for debranching of $\mathrm{K} 6 / \mathrm{K} 48, \mathrm{~K} 11 / \mathrm{K} 48$, and K48/K63 $\mathrm{Ub}_{3}$ by UCH37-RPN13 ${ }^{\mathrm{C}}$ (Figure 2C). Comparison of the $\mathrm{K}_{\mathrm{M}}$ values shows that $\mathrm{UCH} 37-\mathrm{RPN} 13^{\mathrm{C}}$ binds $\mathrm{K} 6 / \mathrm{K} 48$ branched $\mathrm{Ub} 3$ in preference to $\mathrm{K} 11 / \mathrm{K} 48$ or $\mathrm{K} 48 / \mathrm{K} 63$ branched Ub3. Unexpectedly, $k_{\text {cat }}$ was highest for $\mathrm{K} 11 / \mathrm{K} 48 \mathrm{Ub}_{3}$; this resulted in similar catalytic efficiencies $\left(k_{\text {cat }} / K_{\mathrm{M}}\right)$ of debranching by UCH37-RPN13 ${ }^{\mathrm{C}}$ for $\mathrm{K} 6 / \mathrm{K} 48$ and $\mathrm{K} 11 / \mathrm{K} 48 \mathrm{Ub}_{3}$, whereas $\mathrm{K} 48 / \mathrm{K} 63 \mathrm{Ub}_{3}$ is 12 to 16 -times lower. 
We noticed that the $k_{\text {cat }}$ we measured for $\mathrm{K} 6 / \mathrm{K} 48 \mathrm{Ub}_{3}$ debranching at $30{ }^{\circ} \mathrm{C}$ is slower than what was reported by others, which varied from 3 to 12 -fold faster at $37^{\circ} \mathrm{C}$, whereas the $K_{\mathrm{M}}$ values $\operatorname{are~similar}^{29}$. To test if this discrepancy was due to artifacts from affinity tags or different methods of purification, we evaluated four different enzyme preparations by Ub-AMC hydrolysis (Figure S2B) and $\mathrm{Ub}_{3}$ debranching (Figure S2C, D) assays. In neither assay were the results affected significantly by an N-terminal His-TEV tag on UCH37, whether RPN13 ${ }^{\mathrm{C}}$ was added in trans or co-purified with UCH37, or use of full-length RPN13 (RPN13 ${ }^{\mathrm{FL}}$ ) versus RPN13 ${ }^{\mathrm{C}}$.

\section{UCH37-RPN13 ${ }^{C}$ contacts the hydrophobic patches on both distal Ub units in a K6/K48 branched-chain substrate}

The K48-specific debranching by UCH37-RPN13 ${ }^{\mathrm{C}}$ suggests that both distal Ub units in a branched $\mathrm{Ub}_{3}$ substrate make unique contacts with the enzyme. To probe for those interactions, we attached ${ }^{15} \mathrm{~N}$-labeled Ub at either the $\mathrm{K} 6$ or K48 branch and performed NMR studies in the presence of the catalytically-inactive UCH37(C88A)-RPN13 ${ }^{C}$ complex.

For the K48-linked distal Ub, we observed that the enzyme contacts the canonical Ub hydrophobic patch residues L8, 144 and V70 (Figures 3A, S3A), consistent with the reported crystal structures of the UCH37-RPN13 ${ }^{\mathrm{C}}$-Ub complex ${ }^{16,17}$. Additionally, there were notable chemical shift perturbations (CSPs) and signal attenuations in the Ub $\alpha$-helix (residues $23,27,29,30,32-34)$ that did not correspond to any monoUb contacts in the UCH37-RPN13 ${ }^{\mathrm{C}}-\mathrm{Ub}$ crystal structures. For the K6-linked distal Ub, we also observed strong signal attenuations of L8 and 144 (Figures 3B, S3B). The interpretation of these NMR signal perturbations is complicated by the well-known observation that the hydrophobic patch of a proximal Ub in a chain potentially interacts with hydrophobic patch residues of a neighboring distal Ub unit ${ }^{32-34}$. In fact, NMR spectra revealed some effects on the hydrophobic residues in the proximal Ub (Figures 3C, S3C), albeit the signal perturbations were not as severe as in the two distal Ubs. Thus, we sought to simplify the system by eliminating the hydrophobic patch on the proximal Ub. NMR spectra showed that the mutated proximal Ub(L8A,I44A) does not directly interact with UCH37-RPN13 ${ }^{\mathrm{C}}$ (Figure 3D). Importantly, we found that a K6/K48 Ub 3 substrate with 
L8A, I44A mutations in the proximal Ub is debranched by UCH37-RPN13 ${ }^{\mathrm{C}}$ at least as efficiently as the wild-type substrate (Figure $3 \mathrm{E}$ ), indicating that the hydrophobic patch in the proximal $\mathrm{Ub}$ is not required for either binding or catalysis.

\section{The hydrophobic patch on the K6-linked distal $\mathrm{Ub}$ is required for debranching}

To test whether the hydrophobic patch of K6-linked distal Ub is required for the debranching activity, we sought to replace it with a less hydrophobic or charged residue. However, poor utilization of $\mathrm{Ub}(\mathrm{L} 8 \mathrm{~A}, \mathrm{I44A})$ by the $\mathrm{E} 1 / \mathrm{UbcH} 5 / \mathrm{NleL}$ enzymes precluded direct substitution by this Ub mutant. As an alternative approach, we adopted the strategy used by Cooper et al. ${ }^{35} \mathrm{We}$ synthesized K48-linked $\mathrm{Ub}_{2}$ and then attached $\mathrm{Ub}(\mathrm{L} 8 \mathrm{C})$ at $\mathrm{K} 6$ using E1/UbcH5/NleL; Ub(L8C) can be incorporated into chains by the ubiquitination enzymes and then modified with iodoacetic acid to create $S$-carboxymethylcysteine ( $\mathrm{L} 8 \mathrm{Cmc}$ ), thereby adding a negative charge to the side chain. Using these variants of $\mathrm{K} 6 / \mathrm{K}_{4} 8 \mathrm{Ub}_{3}$, we found that $\mathrm{L} 8 \mathrm{C}$ on the K6-linked distal Ub significantly inhibited debranching by UCH37-RPN13'; moreover, debranching was inhibited even further by the $\mathrm{L} 8 \mathrm{Cmc}$ derivative (Figure $4 \mathrm{~A}$ ).

In another approach to ablate the hydrophobic patch interaction, we utilized two variations of a dichloroacetone-based crosslinking strategy. First, $\mathrm{Ub}(\mathrm{G} 76 \mathrm{C})$ was crosslinked to $\mathrm{Ub}-{ }^{48} \mathrm{Ub}(\mathrm{K} 6 \mathrm{C})$ to produce Mimic1 (Figure 4B). The resultant nonhydrolyzable Ub-Ub crosslink is of similar length to a native isopeptide bond but contains an additional carboxylate ${ }^{36}$. To eliminate the carboxylate, in a second variation we used intein chemistry to produce a Ub $b_{75}$-cysteamine derivative, which was then crosslinked to $\mathrm{Ub}-{ }^{48} \mathrm{Ub}(\mathrm{K} 6 \mathrm{C})$ to produce Mimic2 (Figure 4B). Interestingly, while with Mimic1 we observed a 2-fold reduced debranching rate by UCH37RPN13 $^{C}$, Mimic2 was debranched as efficiently as wild-type K6/K48 Ub3 (Figure 4C). We then proceeded to install either wild-type or $\mathrm{Ub}(\mathrm{L} 8 \mathrm{~A}, \mathrm{I44A})$ at $\mathrm{K} 6$ of the proximal Ub using the Mimic2 strategy. Whereas both native $\mathrm{Ub}_{3}$ and wild-type Mimic2 $\mathrm{Ub}_{3}$ were debranched efficiently, the L8A,I44A-containing Mimic2 was completely refractory to UCH37-RPN13C (Figure 4D). As a control, we showed that all the substrates were efficiently hydrolyzed by OTUB1, a K48-specific DUB. 
Taken together, the results of these biochemical experiments demonstrate that UCH37-RPN13C requires hydrophobic patch residues of both distal $\mathrm{Ub}$ units of the branched $\mathrm{Ub}_{3}$ substrate for its debranching activity. These results further support our interpretation of the NMR data as showing that, when bound to $\mathrm{UCH} 37-\mathrm{RPN}^{1} 3^{\mathrm{C}}$, both distal Ub units use their hydrophobic patches to contact the enzyme.

\section{UCH37 activity regulates proteasome condensates upon proteolytic stress}

In order to explore the physiological functions of $\mathrm{UCH} 37$, we generated a UCH37-knockout cell line (KO) by CRISPR from parental (P) HCT116 cells. The KO cell line was then complemented with three versions of $\mathrm{UCH} 37$ : wild-type (WT), catalytically-inactive (C88A), and Ub-binding deficient (E34K, W36D, and I216D; referred to as EWI) (Figures S5A). Successful knockout and reintroduction of UCH37 were confirmed by western blotting (Figures S5B). The EWI mutant was designed based on the crystal structure of the UCH37-RPN13 ${ }^{\mathrm{C}}$-Ub complex (Figure S1C) and its reduced ability to bind Ub was confirmed (Figure S6A).

Osmotic stress was recently shown to trigger the formation of proteasome liquid-liquid phaseseparated (LLPS) condensates that are thought to be centers of active degradation ${ }^{37}$. As expected, upon osmotic stress, UCH37 localized to proteasome foci where K48-linked polyUb was also prominent (Figure 5A). We found that the KO cells accumulated significantly more proteasome foci in comparison with the parental cells (Figure 5B). We also observed increased accumulation of $\mathrm{K} 11 / \mathrm{K} 48$ branched polyUb in the proteasome foci in KO cells (Figure S5C). In comparison with the parental cells, overexpression of WT UCH37 suppressed proteasome foci accumulation, whereas overexpression of C88A or EWI mutant UCH37 had the opposite effects (Figure 5B). Interestingly, although most proteasome foci appeared in the nuclei of the parental cells, there was also a marked increase in cytosolic foci in KO cells (Figure 5C). Similar accumulations of proteasome foci in KO cells were observed following oxidative stress (Figure S5D). These results strongly suggest that UCH37 DUB activity counteracts proteasome foci formation promoted by proteolytic stress. We noticed also that proteasome foci were readily 
detectable in KO cells even without sucrose treatment (Figure 5D), suggesting that the KO cells accumulate proteasome substrates under unperturbed conditions, which is consistent with a recent report ${ }^{38}$. Indeed, analysis of the whole-cell lysates from the KO cells showed accumulation of K48 and K11/K48 polyUb both with and without osmotic stress (Figure 5E and 5F). This phenotype was partially rescued by overexpression of WT UCH37. These results suggest that the loss of $\mathrm{UCH} 37$ negatively impacts proteasome degradation capacity in cells.

\section{Ub, Ub-protein conjugates, and the RAD23B substrate shuttle receptor accumulate on proteasomes containing $\mathrm{UCH} 37(\mathrm{C} 88 \mathrm{~A})$}

Under unperturbed conditions, UCH37 dynamically interacts with the $19 \mathrm{~S} \mathrm{RP}^{39,40}$ and, at steady state, appears in only a minor fraction of isolated mammalian proteasome complexes. To better understand how UCH37 activity affects proteasomal degradation, we focused on UCH37containing proteasomes isolated from WT, C88A, and EWI cells. From the whole-cell lysates, it was apparent that C88A and EWI cells accumulate K48, K11/K48 and, to a lesser extent, total polyUb conjugates; this was the case both with and without osmotic stress (Figure 6A). These results are consistent with the phenotypes observed by proteasome foci counting (Figure 5B). UCH37-containing proteasomes were immunoprecipitated from these cells under low salt conditions to preserve weakly associated proteins (Figure 6B). Whereas the C88A proteasomes accumulated polyUb conjugates, surprisingly, the EWI proteasomes behaved similarly to WT. To confirm these observations, we also created HEK293 cell lines that inducibly express different forms of UCH37 (Figure S6A). Again, polyUb accumulation was only observed with proteasomes containing C88A (lanes 7, 8 and 12). Enhanced polyUb association occurs in the context of the proteasome, not free UCH37, as UCH37 $\triangle$ CTD, which cannot associate with RPN13, did not coimmunoprecipitate with either proteasomes or polyUb conjugates; this was observed with either WT or C88A UCH37 $\triangle$ CTD (Figure S6A, lanes 9 and 10). UCH37 with the EWI and C88A mutations combined had only slightly increased amounts of associated polyUb conjugates in comparison with EWI alone, confirming that the EWI mutations effectively eliminated most, if not all, Ub binding to UCH37 (Figure S6A, lanes 11 and 12). To quantify the amounts and types of (poly)Ub species associated with the UCH37-containing proteasomes, we used Atto532- 
labeled tUI in a previously developed protocol ${ }^{31}$ to inventory free (i.e., unconjugated Ub or unanchored polyUb), activated (i.e., thioester form), and conjugated Ub species (Figure 6C). We found that both free and conjugated Ub species accumulated on C88A proteasomes at a level 4times that of WT or EWI proteasomes. Additionally, the increased polyUb on C88A proteasomes was accompanied by increased association of the substrate shuttle receptor, RAD23B (Figure $6 \mathrm{D})$. These results suggest that, although neither the C88A or EWI form of UCH37 can catalyze deubiquitination, only the C88A proteasomes fail to clear Ub species efficiently.

Taking advantage of its GFP-tag, we examined the dynamics of UCH37 association with proteasomes directly in live cells. After sucrose treatment, we performed Fluorescence Recovery After Photobleaching (FRAP) analyses of GFP-UCH37 in sucrose-induced proteasome foci. Strikingly, the average FRAP recovery half-times for UCH37 WT (10.7 s) and EWI (7.2 s) were 3 to 4 -times shorter than that of C88A (27.4 s) (Figure 6E). Given that the reported $t_{1 / 2}$ of proteasomes in sucrose-induced foci is $13.71 \mathrm{~s}^{37}$, similar to that of UCH37 WT, these data suggest that C88A-proteasomes in the condensates are significantly more static.

To determine the compositions of UCH37-containing proteasomes in an unbiased fashion, we performed Tandem Mass Tag (TMT) mass spectrometry analyses of WT, C88S and EWI proteasomes. For the majority of the constitutive proteasome RP and CP subunits, we did not find any differences (Figure S6E). However, among dynamically associated proteasomeinteracting proteins (PIPs), we found that Ub (UBA52), RAD23B, PA28 (PSME1 and PSME2), USP14, and 19S assembly chaperones (PSMD5 and PSMD10) were noticeably enriched in the C88S and, to a lesser extent, EWI proteasomes (Figure 6F). The extent of Ub accumulation detected by TMT analysis is less than that detected by tUI-based assays (Figure 6C), most likely due to ratio suppression commonly observed in TMT-based quantification ${ }^{41}$. To rule out that this is due to differences between UCH37 C88A and C88S mutants, we compared them in both pulldown assays and in vitro binding assays, and found that both mutants behaved similarly (Figure S6B and S6C). 
The accumulation of RAD23B on C88A (or C88S) proteasomes raised the possibility that, among substrate shuttle proteins, RAD23B may uniquely prefer branched Ub chains; this prompted us to determine the binding affinities between full-length RAD23B and various forms of $\mathrm{Ub}_{3}$. We found that RAD23B does not differentiate linear versus branched $\mathrm{Ub}_{3}$ provided that a K48linkage is present within the chain (Figure S6D).

\section{Discussion}

UCH37 is the only DUB known to date that has a strong preference for branched polyUb chains. Our results have revealed multiple levels of control that contribute to the unique debranching specificity of UCH37. Like other UCH-family of enzymes, the ASCL of UCH37 controls access to its active site. In the ground state, RPN13's interaction with the ASCL promotes a conformation that prevents linear polyUb chains from accessing the active site. By microscale thermophoresis, we determined that $\mathrm{UCH} 37-\mathrm{RPN} 3^{\mathrm{C}}$ bound to $\mathrm{K} 48$ and $\mathrm{K} 6$-linked $\mathrm{Ub}_{2}$ with similarly weak affinities. Additionally, mixed-linkage linear polyUb chains, such as Ub- ${ }^{6} \mathrm{Ub}-{ }^{48} \mathrm{Ub}$ and $\mathrm{Ub}^{48} \mathrm{Ub}-{ }^{6} \mathrm{Ub}$, are poor deubiquitination substrates. This likely reflects that the ASCL is positioned to block linear polyUb binding regardless of linkage. Thus, the ASCL functions as a gate and RPN13 as a gatekeeper to prevent unintended hydrolysis of linear polyUb chains. Upon encountering branched chains, UCH37 engages the hydrophobic patches of both distal Ub units; this would enhance affinity and, crucially, affect the displacement of the ASCL to allow access to the active site. The geometry of a branched polyUb chain likely plays a key role in substrate binding, position of the ASCL, and K48-specificity in debranching by UCH37-RPN13. Our data suggest that the $\mathrm{K} 6 / \mathrm{K} 48$-branched $\mathrm{Ub}_{3}$ presents a favorable configuration to engage and activate the enzyme. Given that we did not observe NMR CSPs of Ub residues around either the K48 linkage or elsewhere on the proximal Ub, we favor a model in which the configuration of the two distal Ubs at the branch point direct specificity for cleavage of the K48 linkage. This model predicts that polyUb substrates with Ub units branching from different lysine residues will not be hydrolyzed with equal efficiencies by UCH37-RPN13; that substrate preference is in 
the order of K6/K48 > K11/K48 >> K63/K48 supports this idea. Currently, we do not know if UCH37-RPN13 is active with branched polyUb lacking K48 linkage.

The minimal branched polyUb chain has three Ub units. Using $\mathrm{K} 6 / \mathrm{K} 48 \mathrm{Ub}_{3}$ as a model, our NMR experiments suggest that $\mathrm{UCH} 37$ has at least two Ub binding sites, each engaging the hydrophobic patch of a distal Ub unit in a branched chain. One of these sites, presumably the S1 site, is occupied in the complex with monoUb described in previous crystal structures ${ }^{16,17}$. These structures showed that the UCH37 UCH domain binds the S1-site Ub through extensive contacts with the classical Ub L8-144-V70 hydrophobic patch as well as the extended C-terminal tail. These interactions are also observed for the K48-linked distal Ub of the K6/K48 $\mathrm{Ub}_{3}$ in our NMR analysis. Unexpectedly, we observed additional perturbations of surface residues in the Ub $\alpha$-helix, most notably K29, D32, and E34, which may represent new interactions with the S1 site that are promoted upon binding the branched chain. Although we have not identified where the second $\mathrm{Ub}$ binding site is on $\mathrm{UCH} 37$, both our NMR analysis and mutagenesis data support that L8 and 144 of the non-K48-linked distal Ub are required for debranching. Additionally, UCH37 might also have contacts with the C-terminal tail of the K6-linked distal Ub. In contrast, debranching did not depend on the hydrophobic patch of the proximal Ub. We also observed significantly smaller perturbations of NMR signals in the proximal Ub upon binding of $\mathrm{K} 6 / \mathrm{K} 48 \mathrm{Ub}_{3}$ to $\mathrm{UCH} 37-\mathrm{RPN} 3^{\mathrm{C}}$, suggesting that the proximal Ub serves primarily to position the distal Ub units rather than interact directly with the enzyme. It is also possible that the proximal $\mathrm{Ub}$ is responsible for displacing the ASCL in concert with binding of the distal Ub units.

The affinities between the several branched $\mathrm{Ub}_{3}$ chains we tested and $\mathrm{UCH} 37-\mathrm{RPN} 13^{\mathrm{C}}$ are generally weak; these were either measured directly as $K_{\mathrm{DS}}$ or inferred from $K_{\mathrm{M}}$ values. Thus, efficient debranching in vivo likely requires additional interactions that bring or retain polyUbprotein substrates on the 26S proteasome. Full-length RPN13 contains an N-terminal PRU domain that can bind $\mathrm{Ub}^{42}$. Although we did not observe differences in activities between $\mathrm{UCH} 37-\mathrm{RPN} 13^{\mathrm{C}}$ and $\mathrm{UCH} 37-\mathrm{RPN} 13^{\mathrm{FL}}$ with a $\mathrm{Ub}_{3}$ substrate, it is possible that longer polyUb chains are required to engage the PRU domain. 
Recently, it was reported that $10-20 \%$ of polyUb chains in cells may contain branches ${ }^{25}$. With a few exceptions ${ }^{27,28,43,44}$, very little is known about the distributions of Ub-Ub linkages in branched polyUb chains, or the identities of the proteins modified by branched forms of polyUb. Upon osmotic or oxidative stresses, we observed cellular inclusions that contain proteasomes and are stained by a K11/K48 bi-specific antibody, suggesting that at least a subset of proteins in those inclusions are modified with branched polyUb chains. Unfortunately, linkage-specific reagents to detect polyUb are very limited, and we were unsuccessful in our attempts to use a K6-linkage specific anti-Ub affimer ${ }^{45}$ to stain cells; thus, we have no evidence as to whether $\mathrm{K} 6 / \mathrm{K} 48$ branched polyUb or K6-linked Ub accumulates in the stress-induced proteasome foci.

In cells lacking $\mathrm{UCH} 37$, the number of proteasome-containing intracellular inclusions are significantly increased, both in the absence and presence of proteolytic stress. This is accompanied by accumulation of K48 and K11/K48-linked polyUb species in the UCH37 KO cells. Overexpression of UCH37 WT not only rescues the KO phenotypes, but further reduces sucroseinduced foci in comparison with the parental cells. In contrast, overexpression of catalyticallyinactive $\mathrm{UCH} 37, \mathrm{C} 88 \mathrm{~A}$ or EWI, increases proteasome foci. We conclude that debranching by UCH37 promotes the dissolution of proteasome degradation centers. Interestingly, when we examined UCH37-containing proteasomes, we found that C88A, but not WT or EWI-containing proteasomes, accumulate polyUb species that include both free and conjugated Ub. Additionally, in comparison with WT or EWI, C88A-proteasomes exhibit much slower association/dissociation kinetics in sucrose-induced foci. These observations suggest that in the absence of debranching activity, UCH37 interactions with the polyUb species lead to their retention at the proteasome, which in turn abnormally retains other dynamic PIPs such as RAD23B and USP14.

Altogether, these results suggest that UCH37 functions to clear polyUb chains, most likely those that contain branches, from the proteasome. It has been shown that branched polyUb can 
promote substrate degradation by enhancing targeting to either p97/VCP or proteasomes ${ }^{27,28}$, although the underlying mechanism is unclear. With $\mathrm{Ub}_{3}$ chains, we did not observe a binding preference for branched over linear chains by RAD23B, as long as a K48-linkage is present, consistent with previous reports ${ }^{46-48}$. Nevertheless, it is possible that endogenous forms of branched polyUb bind proteasomes more tightly by simultaneously engaging multiple Ub receptor sites. Proteasome-associated UCH37 could efficiently disassemble these Ub chains by severing the K48 linkage at branch points, thereby shortening the chains and also promoting chain release because most proteasomal Ub receptors prefer chains with K48 linkages ${ }^{49-51}$. This model of $\mathrm{UCH} 37$ function requires that debranching is slow enough to not compete with substrate targeting, engagement, and the initiation of degradation. For the in vitro degradation of a small model substrate such as titin-127, substrate processing is complete in less than 1 $\min ^{52}$. Thus, with a $k_{\text {cat }}$ of $3.2 \mathrm{~min}^{-1}$ or less, deubiquitination by $\mathrm{UCH} 37$ is unlikely to cause premature release of polyUb-protein substrates from the $26 \mathrm{~S}$ proteasome. This is in contrast to USP14, another proteasome-associated DUB, which exhibits much faster kinetics and can remove the Ub signal prior to substrate commitment. In another scenario, it is also possible that binding to multiple Ub receptor sites on the proteasome slows or stalls substrate translocation; debranching by UCH37 could help to alleviate that problem. These possibilities for UCH37 function are not mutually exclusive. 


\section{Materials and Methods}

\section{Cell culture}

HCT116 cells (ATCC) were maintained in McCoy's 5A modified medium (Hyclone) supplemented with $10 \%$ fetal bovine serum and $1.5 \mathrm{mM}$ glutamine. Flp- $\operatorname{In}^{\mathrm{TM}} \mathrm{T}-\mathrm{REx} \mathrm{x}^{\mathrm{TM}} 293$ Cells (Invitrogen) were cultured in Dulbecco's Modified Eagle's Medium (DMEM, Corning) supplemented with $10 \%$ fetal bovine serum, $2 \mathrm{mM}$ glutamine, and penicillin-streptomycin. All cell lines were kept in a humidified incubator at $37^{\circ} \mathrm{C}$ with $5 \% \mathrm{CO}_{2}$.

\section{Generation of stable cell lines}

UCH37 knockout cells (KO) were generated by CRISPR/Cas9. A pair of gRNAs were used to introduce genomic deletions in the first exon following protocols described by Bauer et al. ${ }^{53}$ Briefly, guide sequences 5'GGCATTGCCCGTCATGGCCC3' and 5'GTCTTCACCGAGCTCATTAA3' were cloned into pX330-U6-Chimeric_BB-CBh-hSpCas9 (a gift from Feng Zhang; Addgene plasmid \# 42230) and co-transfected with a GFP-expressing plasmid into HCT116 cells using Lipofectamine 2000. $48 \mathrm{~h}$ after transfection, single cells with high GFP expression were sorted by flow cytometry. Candidate clones were screened by PCR and successful knockouts were further confirmed by western-blot analysis.

Flag-GFP-tagged UCH37 (WT, C88A, C88S or EWI) were cloned into pQCXIP (Clontech). Retroviruses were produced by co-transfection of pCI-VSVG (a gift from Garry Nolan; Addgene plasmid \#1733) and pQCXIP-UCH37 plasmids into Phoenix-GP cells. $48 \mathrm{~h}$ after transfection, the viral supernatants were harvested, filtered, and added to UCH37 KO cells with $4 \mu \mathrm{g} / \mathrm{ml}$ polybrene. Infected cells were selected with $1 \mu \mathrm{g} / \mathrm{ml}$ puromycin and sorted subsequently by flow cytometry to enrich for low GFP-expression cells.

Flag-tagged UCH37 (WT, C88A, $\triangle \mathrm{CTD}, \triangle \mathrm{CTD} / \mathrm{C} 88 \mathrm{~A}$, EWI, EWI/C88A) were cloned into pcDNA5/FRT/TO (Invitrogen). Stable cell lines that inducibly express these proteins were constructed from Flp-In ${ }^{T M}$ T-REx ${ }^{T M} 293$ Cells following manufacturer's instructions.

\section{Immunoblotting}


Whole cell lysates were obtained by lysing cells in $50 \mathrm{mM}$ Tris- $\mathrm{HCl}, \mathrm{pH}$ 7.6, $10 \mathrm{mM}$ EDTA, 0.5\% SDS, and protease inhibitor cocktail (Sigma P8340), followed by sonication and clarification by centrifugation. After determining protein concentrations by the BCA assay, equal amounts of lysates were loaded and separated by SDS-PAGE using 4-12\% SurePAGE BisTris (GenScript) or 3-8\% NuPAGE Tris-Acetate (ThermoFisher Scientific) gels and then transferred to $0.22 \mu \mathrm{m}$ nitrocellulose membranes. Membranes were subjected to Revert Total Protein Stain (LI-COR), followed by blocking with 5\% non-fat dry milk in PBS-T (0.05\% Tween) for $1 \mathrm{~h}$ at RT before incubation with primary antibodies at $4{ }^{\circ} \mathrm{C}$ overnight. Membranes were then washed with PBS-T three times and further incubated with IRDye secondary antibodies (LICOR Biosciences) for $1 \mathrm{~h}$ at RT. Signals were visualized with a LI-COR Odyssey CLx Imaging System and quantified using Image Studio. Primary antibodies used include: UCH37 (Abcam ab133508, 1:1000); RPN2 (Boston Biochem AP-104, 1:1000), RPN13 (custom made ${ }^{13}, 1: 1000$ ); PSMB5 (Cell Signaling Technology 12919S, 1:1000); ubiquitin (P4D1; Santa Cruz Biotechnology Sc-8017, 1:1000); ubiquitin conjugates (FK2; Enzo Life Sciences PW8810, 1:1000); K48-specific ubiquitin (Millipore 05-1307, 1:2000); K11/K48-bispecific ubiquitin (Genentech, 1:1500); K63specific ubiquitin (Millipore 05-1308, 1:1000); K6 affimer (Avacta AVA00101, 0.1 ug/ml); RAD23B (Santa Cruz Biotechnology sc-67225, 1:1000).

\section{Immunofluorescence and FRAP}

Cells were grown on coverslips to $70-80 \%$ confluency. For proteolytic stresses, HCT116 cells were treated with $0.2 \mathrm{M}$ sucrose or $0.5 \mathrm{mM} \mathrm{NaAsO}_{2}$ for indicated times before they were fixed with 4\% paraformaldehyde in PBS for $15 \mathrm{~min}$ at room temperature and permeabilized with cold methanol for $10 \mathrm{~min}$ at $-20^{\circ} \mathrm{C}$. After washing with PBS, cells were then blocked with $3 \%$ BSA for $1 \mathrm{~h}$ and incubated with primary and secondary antibodies diluted in 1\% BSA, 0.1\% Triton X-100 in PBS. Primary antibodies used include: RPT6 (Enzo Life Sciences PW9265, 1:250); K48-specific ubiquitin (Millipore 05-1307, 1:250); RPN13 (custom made ${ }^{13}, 1: 100$ ) and K11/K48-bispecific ubiquitin (Genentech, 1:1500). Host-specific secondary antibodies conjugated with Alexa Fluor 488/594/647 dyes (Thermo Fisher Scientific) were used. Where needed, nuclei were stained with DAPI and whole cells were stained with HCS CellMask Green (Thermo Fisher Scientific) 
before mounting. Coverslips were mounted onto slides with ProLong Diamond Antifade (Thermo Fisher Scientific).

Images were acquired using a Zeiss LSM 880 confocal microscope with a 63x oil objective /NA1.40. All images were acquired in Z-stacks and maximum projections were used for quantification. Quantification of foci in images was done using CellProfiler v.3.1.954. DAPI signals were used to define nuclei, which were segmented by the Otsu threshold method using a two-classes strategy. HCS CellMask signals were used to define cell boundary with the Watershed method. To identify RPT6 foci within the nuclei, images were segmented by the Robust Background method using a global strategy. The averaging method was set as mean and the variance set at 7 SDs. Statistical analyses were performed using GraphPad Prism v.8. Pairwise analyses used the two-tailed unpaired $\mathrm{t}$ test; the $\mathrm{P}$ values are denoted as $* *(<0.01)$, $* * *(<0.001), * * * *(<0.0001)$. At least 100 cells were counted for each analysis.

FRAP experiments were performed using a Zeiss LSM 880 confocal microscope with 100x oil objective/NA1.46. Acquisition used live HCT116 cells expressing Flag-GFP-UCH37 (WT, C88A or EWI) within $1 \mathrm{~h}$ of treatment with $0.2 \mathrm{M}$ sucrose, on an incubated stage chamber maintained at $37^{\circ} \mathrm{C}$ with a humidified $5 \% \mathrm{CO}_{2}$ atmosphere. Photobleaching of GFP-containing foci was performed with the $488-\mathrm{nm}$ line from a $35 \mathrm{~mW}$ Ar laser operating at $100 \%$ power. The pinhole was set to $2 \mu \mathrm{m}$ and fluorescence recovery was monitored at intervals of $200 \mathrm{~ms}$ using the 488$\mathrm{nm}$ laser line at 1\% power and a GAsP PMT detector. For each cell line, data were obtained from two to five foci per nucleus and 7 or 8 cells. Each bleached spot corresponded to a circle of $1.4 \mu \mathrm{m}$ diameter. Normalized FRAP curves were generated from raw data after background subtraction as described ${ }^{55}$. After normalization, the curves were fit by equations describing a two-exponential fluorescence recovery using GraphPad Prism. The fits were constrained to use a common Fast component while the Slow component for each focus' recovery was allowed to vary.

\section{Expression and purification of recombinant proteins}

His-TEV-UCH37 (WT, C88A or C88S) and GST-TEV-RPN13 ${ }^{\mathrm{C}}$ were expressed in E. coli and purified as described previously ${ }^{16}$. For co-purifications, the harvested UCH37 and RPN13 
(RPN13 ${ }^{\mathrm{C}}$ or RPN13 ${ }^{\mathrm{FL}}$ )- expressing bacterial cell pellets were combined in a 1:1 ratio as described $^{16}$. For NS-UCH37, the pET151-hUCH37(ISF1) plasmid ${ }^{16}$ was modified so that Met1 in $\mathrm{UCH} 37$ is replaced with an N-terminal Ser residue following cleavage by TEV protease. Fulllength RPN13 was expressed from pET19b with an N-terminal 10xHis-tag in Rosetta2 BL21(DE3) cells ${ }^{13}$. Log phase cultures were shifted to $18^{\circ} \mathrm{C}$, induced with $0.4 \mathrm{mM} \mathrm{IPTG}$, and incubation at $18^{\circ} \mathrm{C}$ continued overnight before cells were harvested. His10-Rpn $13^{\mathrm{FL}}$ was purified using HisPur Ni-NTA agarose (Thermo Fisher Scientific) following the manufacturers' instructions. The eluted proteins were further purified by gel filtration (Superdex 200 column; GE Healthcare) in PBS with $5 \mathrm{mM} \beta$-mercaptoethanol. Sequences of different versions of UCH37 proteins are shown in Figure S7.

Human RAD23B was PCR amplified from cDNA and cloned into pGEX-6P between BamHI and Xhol sites. GST-RAD23B was expressed in BL21(DE3) Codon Plus cells grown at $37^{\circ} \mathrm{C}$ to an OD600 of 0.7 , and induced with $0.2 \mathrm{mM} \mathrm{IPTG}$ at $15^{\circ} \mathrm{C}$ for $16 \mathrm{~h}$. Purification was achieved by affinity chromatography (GSTrap FF 5 mL column (GE Healthcare)) in 25 mM Tris, pH7.6, 300 $\mathrm{mM} \mathrm{NaCl}, 2 \mathrm{mM}$ DTT, 1 mM EDTA. The GST tag was removed by Precision protease and RAD23B was buffer exchanged into 50 mM HEPES, pH 7.5, 150 mM NaCl, 1 mM DTT, 10 mM EDTA.

\section{Synthesis of polyubiquitin chains}

Ub and Ub mutants were expressed in Rosetta2 BL21(DE3) cells and purified by established procedures. E1 ${ }^{56}, \mathrm{E} 2$ (UBCH5C $\mathrm{C}^{57}, \mathrm{E} 2-25 \mathrm{~K}^{58}, \mathrm{CDC} 34^{59}, \mathrm{UBE} 2 \mathrm{~S}-\mathrm{UBD}^{60}$ and UBC13/MMS2 ${ }^{61}$ ) and E3 $\left(\mathrm{NleL}^{62}\right)$ enzymes were expressed and purified as described. General strategies and reaction conditions followed those described by Raasi et al $^{63}$ :

- $[\mathrm{Ub}]_{2^{-}}{ }^{6,48} \mathrm{Ub}$ was synthesized by combining $\mathrm{Ub}(\mathrm{K} 6 \mathrm{R}, \mathrm{K} 48 \mathrm{R})$ with $\mathrm{Ub}(\mathrm{D} 77)$ or $\mathrm{Ub}(\Delta \mathrm{GG})$ in a 2:1 molar ratio with $50 \mathrm{nM}$ E1, $10 \mu \mathrm{M}$ UBCH5C and $1 \mu \mathrm{M}$ NleL in the ubiquitination buffer (50 mM Tris-Cl, pH7.5, 5 mM MgCl 2,50 mM NaCl, 0.5 mM EDTA, 0.5 mM DTT, 10 mM ATP, 50 $\mathrm{mM}$ Creatine Phosphate, $3 \mathrm{U} / \mathrm{ml}$ Creatine Kinase, $0.3 \mathrm{U} / \mathrm{ml}$ Pyrophosphatase, 5\% glycerol).

- $[\mathrm{Ub}]_{2}{ }^{11,48} \mathrm{Ub}$ was synthesized by first generating K11-linked $\mathrm{Ub}_{2}$ with $\mathrm{Ub}(\mathrm{K} 11 \mathrm{R}, \mathrm{K} 48 \mathrm{R})$, $\mathrm{Ub}(\mathrm{D} 77)$ or $\mathrm{Ub}(\Delta G G)$ as described ${ }^{60}$. The resulting $\mathrm{Ub}_{2}$ was treated with $\mathrm{AMSH}$, purified by 
cation-exchange chromatography on a Mono S (GE Healthcare) column, then incubated with Ub(K48R), E1 and E2-25K to add the K48-branch.

- $\quad[\mathrm{Ub}]_{2}{ }^{-48,63} \mathrm{Ub}$ was synthesized by first generating K63-linked $\mathrm{Ub}_{2}$ with $\mathrm{Ub}(\mathrm{K} 63 \mathrm{R}, \mathrm{K} 48 \mathrm{R})$ $\mathrm{Ub}(\mathrm{D} 77)$ or $\mathrm{Ub}(\Delta \mathrm{GG})$ as described ${ }^{63}$. After $\mathrm{Ub}_{2}$ was purified by cation-exchange on a Mono $\mathrm{S}$ (GE Healthcare) column, it was incubated with Ub(K48R), E1 and E2-25K to add the K48branch.

- Linear K6, K48 or K63-linked $\mathrm{Ub}_{3}$ were built one $\mathrm{Ub}$ at a time as described ${ }^{63}$. To build K6linked Ub chains, the building blocks all contained the K48R mutation and NleL was used to direct the K6-linkage.

- $[\mathrm{Ub}]_{2^{-}}{ }^{6,48} \mathrm{Ub}$ containing $\mathrm{Ub}(\mathrm{L} 8 \mathrm{C})$ as the K6-linked distal Ub was synthesized by a two-step strategy. First, K48-linked $\mathrm{Ub}_{2}$ was built with $\mathrm{Ub}(\mathrm{K} 6 \mathrm{R}, \mathrm{K} 48 \mathrm{R})$ and $\mathrm{Ub}(\Delta \mathrm{GG})$ using $\mathrm{E} 2-25 \mathrm{~K}$ to direct the K48-linkage. Then $\mathrm{Ub}(\mathrm{K} 6 \mathrm{R}, \mathrm{L} 8 \mathrm{C}, \mathrm{K} 48 \mathrm{R})$ was added to the purified $\mathrm{Ub}_{2}$ and NleL was used to direct the K6-linkage. To further modify the L8C sidechain, $250 \mathrm{mM}$ sodium iodoacetate $\left(\mathrm{pH}^{\sim 7.5}\right)$ was added to the purified $\mathrm{Ub}_{3}$ and incubated in the dark at RT for 30 $\min$. The reaction was quenched with $\beta$-mercaptoethanol and protein product purified over a desalting column.

For NMR studies, K6/K48-branched $\mathrm{Ub}_{3}$ chains were made with domain-specific ${ }^{15} \mathrm{~N}$ isotope labeling in a stepwise manner as detailed elsewhere ${ }^{64}$. For example, to make the $\mathrm{Ub}_{3}$ with ${ }^{15} \mathrm{~N}$ labeled K48-linked distal Ub, we started with 10 mg each of the appropriate chain terminating Ub mutants: ${ }^{15} \mathrm{~N}$-labeled Ub(K6R,K48R) and unlabeled $\mathrm{Ub}(\mathrm{D} 77)$ in a $2 \mathrm{~mL}$ reaction mixture with $0.5 \mu \mathrm{M}$ E1 and $50 \mu \mathrm{M}$ E2-25K, protein breakdown mix ${ }^{63}, 2 \mathrm{mM}$ ATP, and $3 \mathrm{mM}$ TCEP for $12 \mathrm{~h}$ at $30{ }^{\circ} \mathrm{C}$. The $\mathrm{K} 48$-linked $\mathrm{Ub}_{2}$ product was purified by cation-exchange chromatography $(5 \mathrm{~mL}$ HiTrap SP HP column; GE LifeSciences) using a gradient of $\mathrm{NaCl}$ in $50 \mathrm{mM}$ Ammonium acetate, $\mathrm{pH} 4.5$, and then buffer-exchanged into $50 \mathrm{mM}$ Tris, $\mathrm{pH} 8.0$ for the next step of the synthesis. The ${ }^{15} \mathrm{~N}$-labeled K48-linked $\mathrm{Ub}_{2}$ was mixed with unlabeled $\mathrm{Ub}(\mathrm{K} 6 \mathrm{R}, \mathrm{K} 48 \mathrm{R})$ at 1:1 molar ratio in a $2 \mathrm{~mL}$ reaction containing $0.5 \mu \mathrm{M} \mathrm{E} 1,50 \mu \mathrm{M}$ UbcH7 (E2) and $50 \mu \mathrm{M}$ NleL (E3) in the protein breakdown mix, $2 \mathrm{mM}$ ATP, $3 \mathrm{mM}$ TCEP for $12 \mathrm{~h}$ at $30^{\circ} \mathrm{C}$. Cation-exchange chromatography yielded pure K6/K48-linked branched $\mathrm{Ub}_{3}$. Finally, the protein was exchanged into $20 \mathrm{mM}$ 
sodium phosphate buffer, pH 7.2, containing $100 \mathrm{mM} \mathrm{NaCl}, 1 \mathrm{mM}$ TCEP, 1 mM EDTA, and 0.2\% $(\mathrm{w} / \mathrm{v}) \mathrm{NaN}_{3}$. The masses of the $\mathrm{Ub}_{2}$ and $\mathrm{Ub}_{3}$ products were verified by SDS-PAGE and ESI-TOF mass spectrometry. A similar procedure was used to assemble K6/K48-linked branched Ub chains with ${ }^{15} \mathrm{~N}$-labeled K6-linked distal Ub or the proximal Ub.

\section{Synthesis of crosslinked branched Ub chain mimics}

1,3-dichloroacetone (DCA)-based crosslinking was performed essentially as described ${ }^{65}$. For Mimic1, His6-Ub(G76C) and Ub-48 $\mathrm{Ub}(\mathrm{K} 6 \mathrm{C})$ were mixed at equal molar ratio in $50 \mathrm{mM}$ sodium tetraborate, $\mathrm{pH}$ 8.5. $5 \mathrm{mM}$ TCEP was added at RT for $30 \mathrm{~min}$ followed by DCA at an amount equal to one-half of the total sulfhydryl groups in the reaction. After $30 \mathrm{~min}$ on ice, the reaction was quenched with $10 \mathrm{mM} \beta$-mercaptoethanol. Branched $\mathrm{Ub}_{3}$ was purified by gel filtration on Superdex 75 (GE Healthcare). For Mimic2, His6-Ub 75 -mercaptoethylamide was prepared using intein chemistry modified from a previous work ${ }^{66}$. Briefly, His6-Ub 75 (WT or L8A, 144A)-inteinCBD were expressed from pTYB2 plasmids in ER2566 E. coli (New England Biolabs). The expressed fusion proteins were purified on chitin resin and Ub was released by incubating the resin in $100 \mathrm{mM}$ cysteamine at $4{ }^{\circ} \mathrm{C}$ overnight. The resulting His6-Ub 75 -mercaptoethylamide was further purified on Ni-NTA agarose to remove excess cysteamine. The subsequent crosslinking reaction was performed as described for Mimic1.

\section{Deubiquitination Assays}

Purified $\mathrm{UCH} 37$ and $\mathrm{RPN} 13^{\mathrm{C}}$ were mixed at equal molar concentrations and incubated at RT for 15 min before addition into assay buffer (50 mM HEPES, pH 7.5, $50 \mathrm{mM} \mathrm{NaCl}, 2 \mathrm{mM}$ DTT). For gel-based assays, polyubiquitin substrates were added and reactions were incubated at 37 ${ }^{\circ} \mathrm{C}$ with aliquots taken at indicated time points for analysis by SDS-PAGE. Ub-AMC hydrolysis assays were performed as described ${ }^{16}$.

The kinetics of debranching by UCH37-RPN13 ${ }^{C}$ were determined by real-time monitoring of product release with the free Ub sensor, Atto532-tUI ${ }^{31}$. Each reaction contained $500 \mathrm{nM}$ Atto532-tUI, 20 or $40 \mathrm{nM}$ preformed UCH37-RPN13 ${ }^{\mathrm{C}}$ complex, and 0 to $250 \mu \mathrm{M}$ substrates in assay buffer (50 mM HEPES, pH 7.5, $5 \mathrm{mM} \mathrm{DTT,} 50 \mathrm{mM} \mathrm{NaCl}, 0.2 \mathrm{mg} / \mathrm{ml}$ ovalbumin). For 
reactions containing 50,100 , or $250 \mu \mathrm{M}$ substrate, Ub sensor concentration was increased to $1.5 \mu \mathrm{M}$. Reactions at $30^{\circ} \mathrm{C}$ were initiated by enzyme addition and fluorescence was monitored over 500 seconds using a FluoroMax-4 Spectrofluorometer (HORIBA Scientific) with $532 \mathrm{~nm}$ excitation (slit width $4 \mathrm{~nm}$ ) and $553 \mathrm{~nm}$ emission (slit width $3 \mathrm{~nm}$ ). A standard curve was created with known amounts of Ub in order to convert fluorescence increase to free Ub concentration. $V_{0}$ was calculated from initial linear portion of the progress curve. All reactions were done in duplicate. $K_{\mathrm{M}}$ and $\mathrm{k}_{\mathrm{cat}}$ values were determined by nonlinear fits to MichaelisMenten kinetics using GraphPad Prism.

\section{Microscale Thermophoresis (MST)}

MST assays were performed in a Monolith NT.115 using Standard Treated Glass Capillaries (NanoTemper Technologies MO-K002). His-TEV-UCH37(C88S)-RPN13C was labelled with Monolith His-Tag labeling Kit RED-tris-NTA (MO-L008) following manufacturer's instructions. Full-length RAD23B was labeled with the Monolith Protein Labeling kit RED-NHS $2^{\text {nd }}$ Gen (MOL011). Each assay contains 50 nM His-TEV-UCH37(C88S)-RPN13 ${ }^{C}$, or 20 nM RAD23B, and varied polyUb concentrations. The assays were performed in PBS containing $0.05 \%$ Tween-20, at 80 or $100 \%$ of excitation power, and $40 \%$ of MST power. MST traces were analyzed at 5 seconds. 1 mM DTT was supplemented to assays with RAD23B. Change of fluorescence, $F_{\text {norm } \% \text {, was }}$ plotted against polyUb concentration and the curves were fitted with a single-site binding model to determine the binding affinity $\left(K_{D}\right)$ using GraphPad Prism.

\section{Immunoprecipitation}

Cells from one $10 \mathrm{~cm}$ dish were washed with PBS, harvested, and resuspended in $350 \mu \mathrm{L}$ low salt lysis buffer (20 mM HEPES, $50 \mathrm{mM} \mathrm{NaCl}, 10 \mathrm{mM} \mathrm{KCl}, 1.5 \mathrm{mM} \mathrm{MgCl}_{2}, 10 \%$ glycerol, 0.5\% Triton X-100, pH 7.9) supplemented with $10 \mathrm{mM}$ iodoacetamide and protease inhibitors (Sigma P8340) on ice for $30 \mathrm{~min}$. Lysates were cleared by centrifugation and incubated with $30 \mu \mathrm{l}$ antiFlag agarose (Sigma A2220) at $4^{\circ} \mathrm{C}$ overnight with rotation. Beads were washed three times with lysis buffer and bound proteins were eluted with 2x Laemmli sample buffer and analyzed by immunoblotting. Alternatively, to quantify the (poly)Ub species, bound proteins were eluted 
with $0.2 \mathrm{mg} / \mathrm{ml}$ 3xFlag peptide. Total, free, activated, and conjugated Ub species were determined using Atto532-labeled tUI following a previously described protocol ${ }^{31}$.

\section{Mass spectrometry analysis of UCH37-containing proteasomes}

Native proteasomes were purified according to Wang et $\mathrm{al}^{40}$. Briefly, HCT116 cells from $5 \times 15 \mathrm{~cm}$ dishes were washed once with PBS and crosslinked in $0.025 \%$ formaldehyde in PBS for $10 \mathrm{~min}$ at $37^{\circ} \mathrm{C}$, followed by the addition of $0.125 \mathrm{M}$ glycine to quench the crosslinker. After washes, cells were harvested and lysed in lysis buffer $(50 \mathrm{mM} \mathrm{NaPi}, \mathrm{pH} 7.5,100 \mathrm{mM} \mathrm{NaCl}, 10 \%$ glycerol, 0.5\% NP-40, $5 \mathrm{mM} \mathrm{MgCl}$, $5 \mathrm{mM}$ ATP, $1 \mathrm{mM}$ DTT) supplemented with protease inhibitors (Sigma P8340) and phosphatase inhibitors (GoldBio GB-450). Lysates were passed through a $21 \mathrm{G}$ needle 20 times, incubated on ice for $15 \mathrm{~min}$, then centrifuged at 18,000 x $\mathrm{g}$ for $15 \mathrm{~min}$ at $4^{\circ} \mathrm{C}$. The supernatant was incubated with anti-GFP nanobody crosslinked to Sepharose ${ }^{67}$ for $2 \mathrm{~h}$ at $4{ }^{\circ} \mathrm{C}$ with rotation. The beads were washed twice with lysis buffer and once with wash buffer ( $25 \mathrm{mM} \mathrm{NaPi}$, pH 7.5, $150 \mathrm{mM} \mathrm{NaCl}, 5 \mathrm{mM} \mathrm{ATP}, 5 \%$ glycerol), then resuspended in $25 \mathrm{mM}$ ammonium bicarbonate, $5 \mathrm{mM}$ TCEP, $8 \mathrm{M}$ urea, $10 \mathrm{mM}$ iodoacetamide, incubated in dark at RT for 30 min. After dilution with 25 mM ammonium bicarbonate, on-bead digestions were done with $2 \mu \mathrm{g}$ LysC (Wako chemicals 125-05061) in $4 \mathrm{M}$ urea and $4 \mu \mathrm{g}$ trypsin (Promega ADV5113) in 1.2 M urea sequentially before quenching with $1 \%$ formic acid. Peptides were collected and combined with multiple washes of the beads with $0.1 \%$ formic acid in $25 \%$ acetonitrile (ACN).

For tandem mass tag (TMT) labeling, individual digested peptide mixtures were C18 cleaned using Waters C18 Sep-PAK cartridges and vacuum concentrated. They were then diluted using 50mM TEAB (triethyl ammonium bicarbonate) and adjusted to $\mathrm{pH} \sim 8$ through multiple cycles of dilution with water and TEAB and vacuum concentration. The final $50 \mu \mathrm{L}$ mixtures were individually labeled using $20 \mu \mathrm{g}$ of a single channel of TMT10plex isobaric labeling reagent (Thermo Fisher Scientific PI90110) in anhydrous ACN and incubated for $1 \mathrm{~h}$ at RT. Hydroxylamine was added to each sample to a final concentration of $0.25 \%$ and incubated

for 15 min with occasional vortexing to quench the labeling reaction. Samples were cleaned and desalted again using Waters C18 Sep-PAK cartridges to remove excess TMT labeling reagent 
and vacuum concentrated. All 10 channels of TMT10plex-labeled samples were combined and analyzed in triplicate by LC MS/MS utilizing a Thermo Scientific ${ }^{\text {TM }}$ EASY-nLC ${ }^{\text {TM }} 1000$ UPLC system coupled on-line to a Thermo Scientific ${ }^{\mathrm{TM}}$ Orbitrap Fusion Lumos ${ }^{\mathrm{TM}}$ Mass Spectrometer. A Thermo Scientific ${ }^{\mathrm{TM}}$ EASY-Spray ${ }^{\mathrm{TM}}$ source with a $25 \mathrm{~cm} \times 75 \mu \mathrm{m}$ PepMap EASY-Spray Column was used to separate peptides over a 90 min gradient of $6 \%$ to $35 \%$ ACN in $0.1 \%$ formic acid at a flow rate of $300 \mathrm{~nL} / \mathrm{min}$. $\mathrm{MS}^{1}$ and $\mathrm{MS}^{2}$ scans were both acquired in the Orbitrap. For MS1 scans, the scan range was set from 375 to $1500 \mathrm{~m} / \mathrm{z}$, resolution set to 12,000 , and the AGC target set to $1 \times 10^{6}$. For $\mathrm{MS}^{2}$ scans, the resolution was set to 50,000 , the $\mathrm{AGC}$ target was set to $1 \times 10^{5}$, the precursor isolation width was $0.8 \mathrm{~m} / \mathrm{z}$, and the maximum injection time was $110 \mathrm{~ms}$. The HCD MS/MS normalized collision energy (NCE) was set to $38 \%$.

Thermo Scientific Proteome Discoverer 2.3 software with SEQUEST ${ }^{\circledR}$ was used for protein identification against a database containing all SwissProt entries for Homo sapiens (February, 2020). Searches were performed using a $10 \mathrm{ppm}$ precursor ion tolerance, the product ion tolerance was set to $0.1 \mathrm{Da}$. TMT tags on lysine residues and peptide $\mathrm{N}$ termini (+229.163 Da) and carboxyamidomethylation of cysteine residues $(+57.021 \mathrm{Da})$ were set as static modifications, while oxidation of methionine residues (+15.995 Da) was set as a variable modification. Peptide-spectrum matches (PSMs) and protein false discovery rates (FDRs) were set as $1 \%$. Reporter ion intensities were adjusted to correct for the isotopic impurities of the different TMT reagents according to manufacturer's specifications.

To compare UCH37 (WT, C88S or EWI)-containing proteasomes, the abundance of each identified protein was first normalized against those in the WT sample. We then used the average abundance of the $19 \mathrm{~S}$ complex subunits as a measure of the amount of proteasomes in each sample. This allowed us to compare the relative quantities of proteasome subunits and proteasome-interacting proteins (PIPs) on a per-proteasome basis.

\section{NMR experiments and chemical shift perturbation mapping}

All samples for NMR measurements were prepared in $20 \mathrm{mM}$ sodium phosphate buffer with pH7.2 containing $100 \mathrm{mM} \mathrm{NaCl} 1 \mathrm{mM}$ TCEP, $1 \mathrm{mM}$ EDTA, $0.2 \% \mathrm{NaN}_{3}$, and 10\% $\mathrm{D}_{2} \mathrm{O}$. The NMR measurements were performed at $23^{\circ} \mathrm{C}, 30^{\circ} \mathrm{C}$, and $37^{\circ} \mathrm{C}$ on an Avance III $600 \mathrm{MHz}$ Bruker 
NMR spectrometer equipped with a cryoprobe. The data were processed using Topspin 3.6.3 (Bruker) and analyzed using Sparky 3.114 ${ }^{68}$.

Binding studies by NMR were done by adding pre-calculated amounts of unlabeled co-purified $\mathrm{UCH} 37(\mathrm{C} 88 \mathrm{~A})-\mathrm{RPN} 13^{\mathrm{C}}$ to ${ }^{15} \mathrm{~N}$-labeled $\mathrm{Ub}_{3}$ or $\mathrm{Ub}_{1}$ up to $1.2: 1$ molar ratio and monitoring changes in $2 \mathrm{D}^{1} \mathrm{H}-{ }^{15} \mathrm{~N}$ SOFAST-HMQC spectra recorded at every titration point. The starting concentration of $\mathrm{Ub}_{3}$ or $\mathrm{Ub}_{1}$ was $100 \mu \mathrm{M}$, the UCH37-RPN13 ${ }^{\mathrm{C}}$ stock concentration was $268 \mu \mathrm{M}$. Changes in amide peak positions in ${ }^{1} \mathrm{H}-{ }^{15} \mathrm{~N}$ NMR spectra were quantified as chemical shift perturbations (CSPS) using the following equation: CSP $=\left[\left(\Delta \delta_{H}\right)^{2}+\left(\Delta \delta_{N} / 5\right)^{2}\right]^{1 / 2}$, where $\Delta \delta_{H}$ and

$\Delta \delta_{N}$ are the chemical shift differences for ${ }^{1} \mathrm{H}$ and ${ }^{15} \mathrm{~N}$, respectively, for a given residue between the free protein and upon addition of UCH37-RPN13 ${ }^{\mathrm{C}}$. Signal attenuations were quantified as the ratio of NMR signal intensities, $I / I_{0}$, in the spectra measured upon $(I)$ and prior to $\left(I_{0}\right)$ addition of the ligand. To compensate for the overall reduction of the NMR signals caused by increased size (slower tumbling) of $\mathrm{Ub}_{3}\left(26 \mathrm{kDa}\right.$ ) upon complex formation with UCH37-RPN13 ${ }^{\mathrm{C}}$ $(52 \mathrm{KDa})$, the final spectra at the end of titration were recorded with a higher number of scans (see Figure S3), and the resulting intensities ( $/$ and $/ 0$ ) were divided by the respective number of scans. The $I / I_{0}$ ratio was further corrected by the volume dilution factor.

\section{Acknowledgements}

We thank Melanine Furgason and Andrew Roddam for the initial development of the Mimic2 Ub conjugation strategy, and to Yun-Seok Choi for materials and advice for the real-time deubiquitination assay with tUI. This work was supported by NIH grants R01 GM098401 to T.Y., R01 GM115997 and R21GM135818 to R.E.C., and R01 GM065334 to D. F.

\section{Competing Interests}

The authors declare no competing interests. 


\section{REFERENCES}

1. Lee, M.J., Lee, B.H., Hanna, J., King, R.W. \& Finley, D. Trimming of ubiquitin chains by proteasome-associated deubiquitinating enzymes. Mol Cell Proteomics 10, R110 003871 (2011).

2. Yao, T. \& Cohen, R.E. A cryptic protease couples deubiquitination and degradation by the proteasome. Nature 419, 403-7 (2002).

3. Verma, R. et al. Role of Rpn11 metalloprotease in deubiquitination and degradation by the 265 proteasome. Science 298, 611-5 (2002).

4. Worden, E.J., Dong, K.C. \& Martin, A. An AAA Motor-Driven Mechanical Switch in Rpn11 Controls Deubiquitination at the 26S Proteasome. Mol Cell 67, 799-811 e8 (2017).

5. Leggett, D.S. et al. Multiple associated proteins regulate proteasome structure and function. Mol Cell 10, 495-507 (2002).

6. Lee, B.H. et al. USP14 deubiquitinates proteasome-bound substrates that are ubiquitinated at multiple sites. Nature 532, 398-401 (2016).

7. Lee, B.H. et al. Enhancement of proteasome activity by a small-molecule inhibitor of USP14. Nature 467, 179-84.

8. Hanna, J. et al. Deubiquitinating enzyme Ubp6 functions noncatalytically to delay proteasomal degradation. Cell 127, 99-111 (2006).

9. Bashore, C. et al. Ubp6 deubiquitinase controls conformational dynamics and substrate degradation of the 26 S proteasome. Nat Struct Mol Biol 22, 712-9 (2015).

10. Li, J. et al. Capzimin is a potent and specific inhibitor of proteasome isopeptidase Rpn11. Nat Chem Biol 13, 486-493 (2017).

11. D'Arcy, P. et al. Inhibition of proteasome deubiquitinating activity as a new cancer therapy. Nat Med 17, 1636-40 (2011).

12. Hamazaki, J. et al. A novel proteasome interacting protein recruits the deubiquitinating enzyme UCH37 to $26 \mathrm{~S}$ proteasomes. EMBO J 25, 4524-36 (2006).

13. Yao, T. et al. Proteasome recruitment and activation of the Uch37 deubiquitinating enzyme by Adrm1. Nat Cell Biol 8, 994-1002 (2006).

14. Qiu, X.B. et al. hRpn13/ADRM1/GP110 is a novel proteasome subunit that binds the deubiquitinating enzyme, UCH37. EMBO J 25, 5742-53 (2006).

15. Yao, T. et al. Distinct modes of regulation of the Uch37 deubiquitinating enzyme in the proteasome and in the Ino80 chromatin-remodeling complex. Mol Cell 31, 909-17 (2008).

16. VanderLinden, R.T. et al. Structural basis for the activation and inhibition of the UCH37 deubiquitylase. Mol Cell 57, 901-911 (2015).

17. Sahtoe, D.D. et al. Mechanism of UCH-L5 activation and inhibition by DEUBAD domains in RPN13 and INO80G. Mol Cell 57, 887-900 (2015).

18. Al-Shami, A. et al. Regulators of the proteasome pathway, Uch37 and Rpn13, play distinct roles in mouse development. PLoS One 5, e13654 (2010).

19. Wicks, S.J. et al. The deubiquitinating enzyme UCH37 interacts with Smads and regulates TGF-beta signalling. Oncogene 24, 8080-4 (2005).

20. Zhou, Z. et al. The deubiquitinase UCHL5/UCH37 positively regulates Hedgehog signaling by deubiquitinating Smoothened. J Mol Cell Biol 10, 243-257 (2018). 
21. Nishi, R. et al. Systematic characterization of deubiquitylating enzymes for roles in maintaining genome integrity. Nat Cell Biol 16, 1016-26, 1-8 (2014).

22. Randles, L., Anchoori, R.K., Roden, R.B. \& Walters, K.J. The Proteasome Ubiquitin Receptor hRpn13 and Its Interacting Deubiquitinating Enzyme Uch37 Are Required for Proper Cell Cycle Progression. J Biol Chem 291, 8773-83 (2016).

23. $\mathrm{Li}, \mathrm{Z}$. et al. Proteasomal deubiquitinase $\mathrm{UCH} 37$ inhibits degradation of beta-catenin and promotes cell proliferation and motility. Acta Biochim Biophys Sin (Shanghai) 51, 277284 (2019).

24. Jacobson, A.D., MacFadden, A., Wu, Z., Peng, J. \& Liu, C.W. Autoregulation of the 26S proteasome by in situ ubiquitination. Mol Biol Cell 25, 1824-35 (2014).

25. Swatek, K.N. et al. Insights into ubiquitin chain architecture using Ub-clipping. Nature 572, 533-537 (2019).

26. Haakonsen, D.L. \& Rape, M. Branching Out: Improved Signaling by Heterotypic Ubiquitin Chains. Trends Cell Biol 29, 704-716 (2019).

27. Yau, R.G. et al. Assembly and Function of Heterotypic Ubiquitin Chains in Cell-Cycle and Protein Quality Control. Cell 171, 918-933 e20 (2017).

28. Meyer, H.J. \& Rape, M. Enhanced protein degradation by branched ubiquitin chains. Cell 157, 910-21 (2014).

29. Deol, K.K. et al. Proteasome-Bound UCH37/UCHL5 Debranches Ubiquitin Chains to Promote Degradation. Mol Cell 80, 796-809 e9 (2020).

30. Sanchez-Pulido, L., Kong, L. \& Ponting, C.P. A common ancestry for BAP1 and Uch37 regulators. Bioinformatics 28, 1953-6 (2012).

31. Choi, Y.S. et al. High-affinity free ubiquitin sensors for quantifying ubiquitin homeostasis and deubiquitination. Nat Methods 16, 771-777 (2019).

32. Cook, W.J., Jeffrey, L.C., Carson, M., Chen, Z. \& Pickart, C.M. Structure of a diubiquitin conjugate and a model for interaction with ubiquitin conjugating enzyme (E2). J Biol Chem 267, 16467-71 (1992).

33. Varadan, R., Walker, O., Pickart, C. \& Fushman, D. Structural properties of polyubiquitin chains in solution. J Mol Biol 324, 637-47 (2002).

34. Virdee, S., Ye, Y., Nguyen, D.P., Komander, D. \& Chin, J.W. Engineered diubiquitin synthesis reveals Lys29-isopeptide specificity of an OTU deubiquitinase. Nat Chem Biol 6, 750-7 (2010).

35. Cooper, E.M. et al. K63-specific deubiquitination by two JAMM/MPN+ complexes: BRISC-associated Brcc36 and proteasomal Poh1. EMBO J 28, 621-31 (2009).

36. Yin, L., Krantz, B., Russell, N.S., Deshpande, S. \& Wilkinson, K.D. Nonhydrolyzable diubiquitin analogues are inhibitors of ubiquitin conjugation and deconjugation. Biochemistry 39, 10001-10 (2000).

37. Yasuda, S. et al. Stress- and ubiquitylation-dependent phase separation of the proteasome. Nature 578, 296-300 (2020).

38. Osei-Amponsa, V. et al. Impact of Losing hRpn13 Pru or UCHL5 on Proteasome Clearance of Ubiquitinated Proteins and RA190 Cytotoxicity. Mol Cell Biol 40(2020).

39. Wang, X. \& Huang, L. Identifying dynamic interactors of protein complexes by quantitative mass spectrometry. Mol Cell Proteomics 7, 46-57 (2008). 
40. Wang, X. et al. Mass spectrometric characterization of the affinity-purified human 26S proteasome complex. Biochemistry 46, 3553-65 (2007).

41. Ting, L., Rad, R., Gygi, S.P. \& Haas, W. MS3 eliminates ratio distortion in isobaric multiplexed quantitative proteomics. Nat Methods 8, 937-40 (2011).

42. Schreiner, P. et al. Ubiquitin docking at the proteasome through a novel pleckstrinhomology domain interaction. Nature 453, 548-52 (2008).

43. Kaiho-Soma, A. et al. TRIP12 promotes small-molecule-induced degradation through K29/K48-branched ubiquitin chains. Mol Cell 81, 1411-1424 e7 (2021).

44. Ohtake, F., Tsuchiya, H., Saeki, Y. \& Tanaka, K. K63 ubiquitylation triggers proteasomal degradation by seeding branched ubiquitin chains. Proc Natl Acad Sci U S A 115, E1401E1408 (2018).

45. Michel, M.A., Swatek, K.N., Hospenthal, M.K. \& Komander, D. Ubiquitin Linkage-Specific Affimers Reveal Insights into K6-Linked Ubiquitin Signaling. Mol Cell 68, 233-246 e5 (2017).

46. Varadan, R., Assfalg, M., Raasi, S., Pickart, C. \& Fushman, D. Structural determinants for selective recognition of a Lys48-linked polyubiquitin chain by a UBA domain. Mol Cell 18, 687-98 (2005).

47. Boughton, A.J., Krueger, S. \& Fushman, D. Branching via K11 and K48 Bestows Ubiquitin Chains with a Unique Interdomain Interface and Enhanced Affinity for Proteasomal Subunit Rpn1. Structure 28, 29-43 e6 (2020).

48. Nakasone, M.A., Livnat-Levanon, N., Glickman, M.H., Cohen, R.E. \& Fushman, D. Mixedlinkage ubiquitin chains send mixed messages. Structure 21, 727-40 (2013).

49. Shi, Y. et al. Rpn1 provides adjacent receptor sites for substrate binding and deubiquitination by the proteasome. Science 351(2016).

50. Lu, X., Ebelle, D.L., Matsuo, H. \& Walters, K.J. An Extended Conformation for K48 Ubiquitin Chains Revealed by the hRpn2:Rpn13:K48-Diubiquitin Structure. Structure 28, 495-506 e3 (2020).

51. Tsuchiya, H. et al. In Vivo Ubiquitin Linkage-type Analysis Reveals that the Cdc48Rad23/Dsk2 Axis Contributes to K48-Linked Chain Specificity of the Proteasome. Mol Cell 66, 488-502 e7 (2017).

52. Bard, J.A.M., Bashore, C., Dong, K.C. \& Martin, A. The 265 Proteasome Utilizes a Kinetic Gateway to Prioritize Substrate Degradation. Cell 177, 286-298 e15 (2019).

53. Bauer, D.E., Canver, M.C. \& Orkin, S.H. Generation of genomic deletions in mammalian cell lines via CRISPR/Cas9. J Vis Exp, e52118 (2015).

54. McQuin, C. et al. CellProfiler 3.0: Next-generation image processing for biology. PLoS Biol 16, e2005970 (2018).

55. McNally, J.G. Quantitative FRAP in analysis of molecular binding dynamics in vivo. Methods Cell Biol 85, 329-51 (2008).

56. Berndsen, C.E. \& Wolberger, C. A spectrophotometric assay for conjugation of ubiquitin and ubiquitin-like proteins. Anal Biochem 418, 102-10 (2011).

57. Lorick, K.L., Jensen, J.P. \& Weissman, A.M. Expression, purification, and properties of the Ubc4/5 family of E2 enzymes. Methods Enzymol 398, 54-68 (2005). 
58. Haldeman, M.T., Xia, G., Kasperek, E.M. \& Pickart, C.M. Structure and function of ubiquitin conjugating enzyme E2-25K: the tail is a core-dependent activity element. Biochemistry 36, 10526-37 (1997).

59. Choi, Y.S. et al. The human Cdc34 carboxyl terminus contains a non-covalent ubiquitin binding activity that contributes to SCF-dependent ubiquitination. J Biol Chem 285, 17754-62 (2010).

60. Bremm, A. \& Komander, D. Synthesis and analysis of K11-linked ubiquitin chains. Methods Mol Biol 832, 219-28 (2012).

61. Hofmann, R.M. \& Pickart, C.M. In vitro assembly and recognition of Lys-63 polyubiquitin chains. J Biol Chem 276, 27936-43 (2001).

62. Lin, D.Y., Diao, J., Zhou, D. \& Chen, J. Biochemical and structural studies of a HECT-like ubiquitin ligase from Escherichia coli 0157:H7. J Biol Chem 286, 441-9 (2011).

63. Raasi, S. \& Pickart, C.M. Ubiquitin chain synthesis. Methods Mol Biol 301, 47-55 (2005).

64. Ranjani, V., Assfalg, M. \& Fushman, D. Using NMR spectroscopy to monitor ubiquitin chain conformation and interactions with ubiquitin-binding domains. Methods Enzymol 399, 177-92 (2005).

65. Long, L., Furgason, M. \& Yao, T. Generation of nonhydrolyzable ubiquitin-histone mimics. Methods 70, 134-8 (2014).

66. Wilkinson, K.D., Gan-Erdene, T. \& Kolli, N. Derivitization of the C-Terminus of Ubiquitin and Ubiquitin-like Proteins Using Intein Chemistry: Methods and Uses. Methods Enzymol 399, 37-51 (2005).

67. Schellenberg, M.J., Petrovich, R.M., Malone, C.C. \& Williams, R.S. Selectable high-yield recombinant protein production in human cells using a GFP/YFP nanobody affinity support. Protein Sci 27, 1083-1092 (2018).

68. Goddard, T.D. \& Kneller, D.G. Sparky 3. University of California, San Francisco (2002). 
A

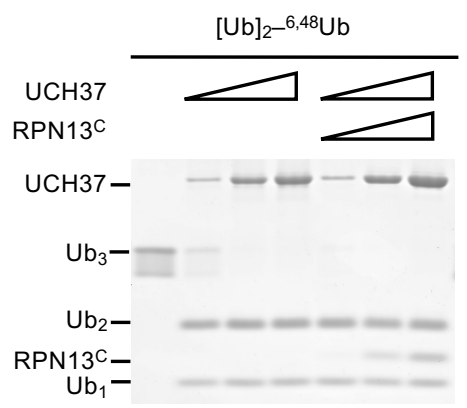

B

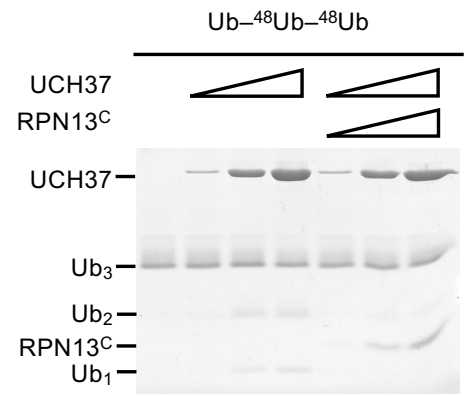

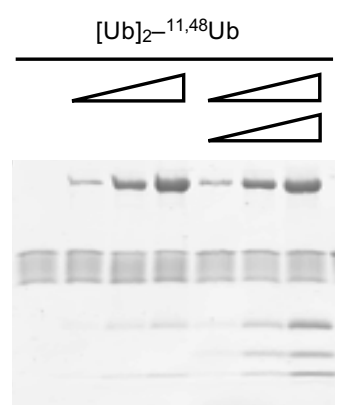
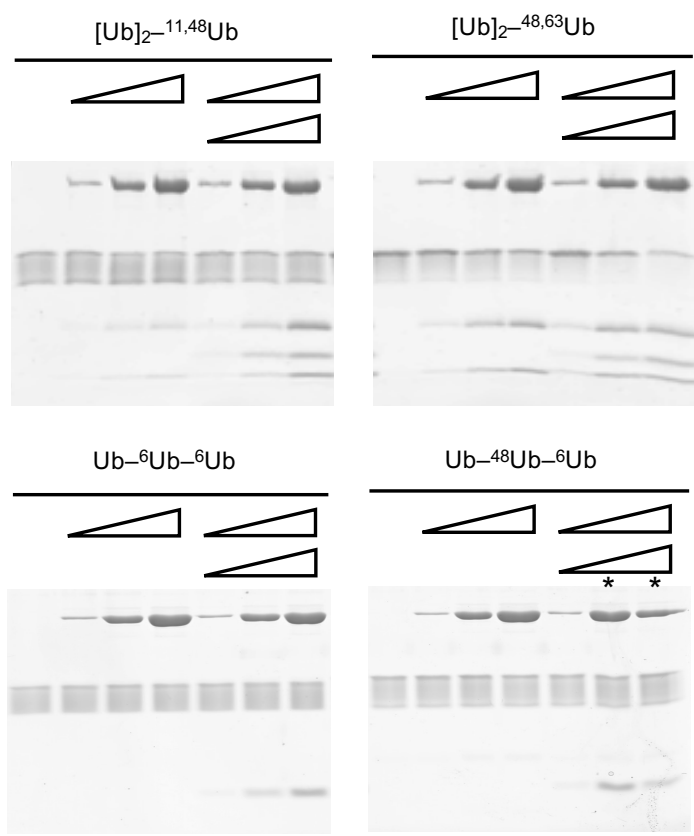
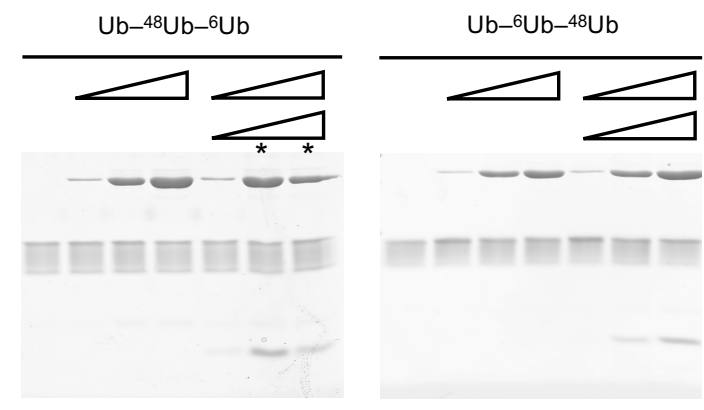

C

UCH37

UCH37-RPN13C
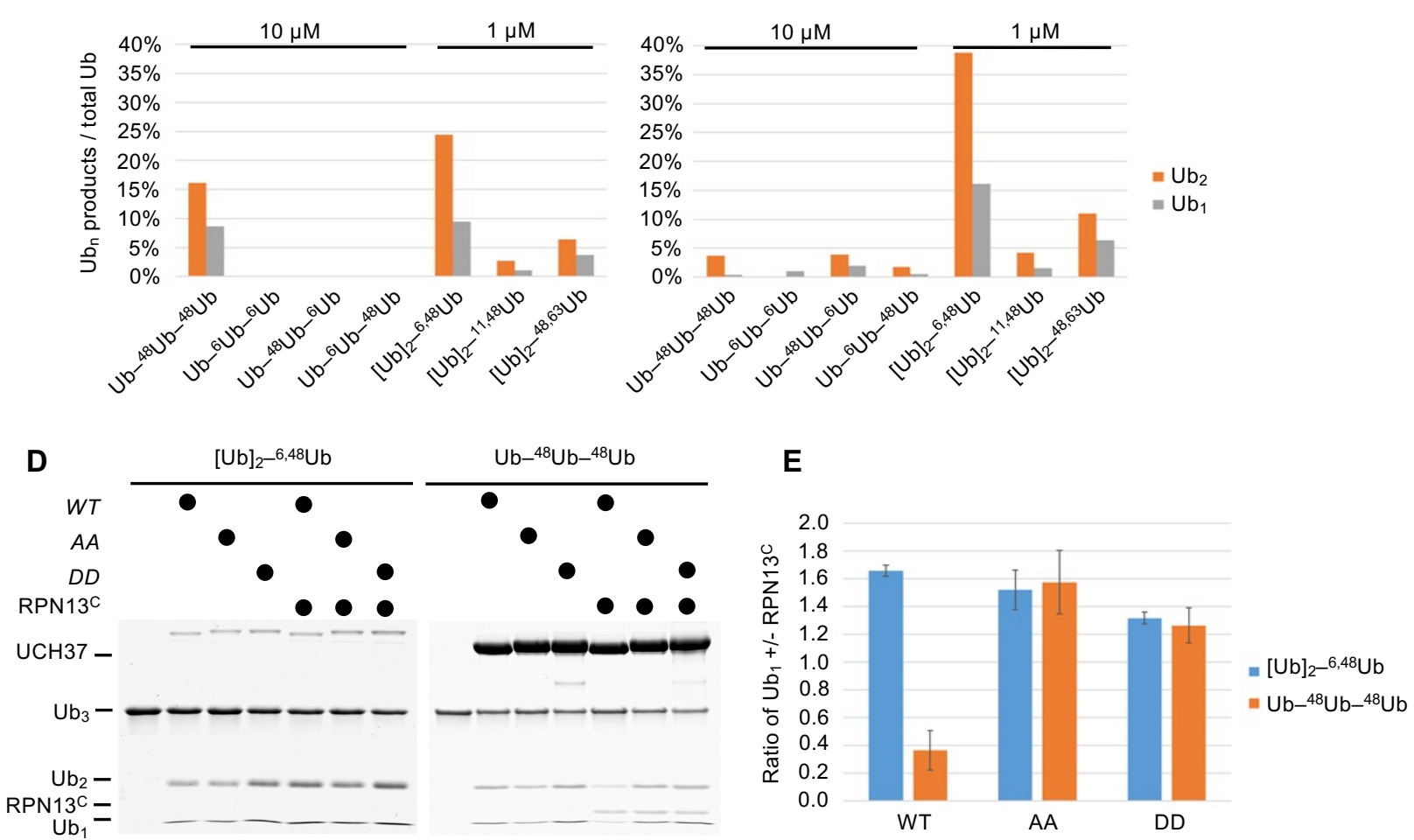

Figure 1. UCH37-RPN13C preferentially cleaves branched polyUb chains. Branched (A) or linear $(B) \mathrm{Ub}_{3}$ substrates $(5 \mu \mathrm{M})$ with the indicated $\mathrm{Ub}-\mathrm{Ub}$ linkages were incubated for $1 \mathrm{~h}$ at $37^{\circ} \mathrm{C}$ with 1,5 , or $10 \mu \mathrm{M}$ His-TEV$\mathrm{UCH} 37$, with or without the addition of equimolar RPN13C. Reactions were analyzed by SDS-PAGE and Coomassie staining. (C) Quantification of the $\mathrm{Ub}_{2}$ and $\mathrm{Ub}_{1}$ products from (A) and (B). For linear $\mathrm{Ub}_{3}$ substrates, results from incubations with $10 \mu \mathrm{M}$ enzyme are shown; for branched $\mathrm{Ub}_{3}$ substrates, results from $1 \mu \mathrm{M}$ enzyme are shown. (D) Wild-type $(W T)$, M148A F149A $(A A)$, or M148D F149D $(D D) \mathrm{UCH} 37$, alone or with the addition of equimolar of RPN13, were incubated with $\mathrm{Ub}_{3}$ substrates for $2 \mathrm{~h}$ at $37^{\circ} \mathrm{C}$. Left, reactions contained $0.5 \mu \mathrm{M}$ enzymes and $10 \mu \mathrm{M}$ branched substrates. Right, reactions contained $10 \mu \mathrm{M}$ enzymes and $5 \mu \mathrm{M}$ linear $\mathrm{Ub}_{3}$. (E) Quantification from (D), plotted as the ratio of $\mathrm{Ub}_{1}$ produced in the presence over absence of RPN13C. Mean \pm SD from two independent replicates are shown. 


\section{A}

\begin{tabular}{|c|c|c|c|}
\hline & Ub chains & $\begin{array}{l}\text { Proximal Ub } \\
\text { modification }\end{array}$ & $K_{\mathrm{D}}(\mu \mathrm{M})$ \\
\hline \multirow[t]{5}{*}{ Linear } & Ub-6Ub & D77 & $42.2 \pm 0.6$ \\
\hline & Ub-6 Ub-6 $U b$ & D77 & $50.0 \pm 4.1$ \\
\hline & Ub-48Ub & - & $63.3 \pm 1.7$ \\
\hline & Ub_-48Ub_48Ub & - & $25.5 \pm 2.4$ \\
\hline & Ub_-63Ub-63Ub & - & $56.9 \pm 2.3$ \\
\hline \multirow[t]{4}{*}{ Branched } & {$[\mathrm{Ub}]_{2}-^{6,48} \mathrm{Ub}$} & D77 & $4.9 \pm 0.2$ \\
\hline & {$[\mathrm{Ub}]_{2}-^{6,48} \mathrm{Ub}$} & $\Delta G G$ & $4.7 \pm 0.2$ \\
\hline & {$[\mathrm{Ub}]_{2}-11,48 \mathrm{Ub}$} & D77 & $43.4 \pm 1.4$ \\
\hline & {$[\mathrm{Ub}]_{2}-48,63 \mathrm{Ub}$} & D77 & $40.2 \pm 1.3$ \\
\hline
\end{tabular}

B
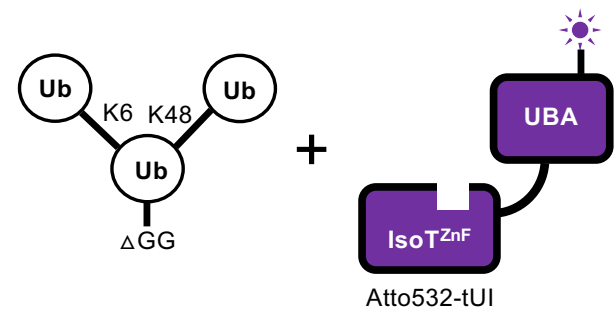

C

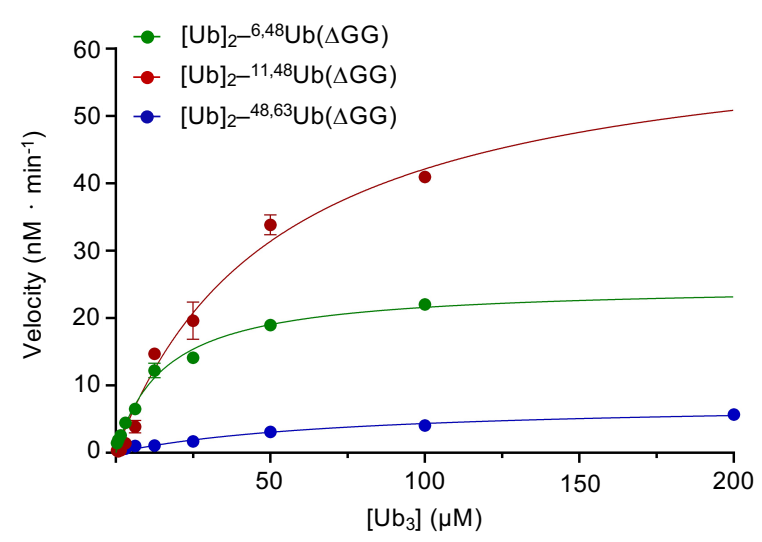

\begin{tabular}{cccc}
\hline & $K_{\mathrm{M}}(\mu \mathrm{M})$ & $\mathbf{k}_{\mathrm{cat}}\left(\mathrm{min}^{-1}\right)$ & $\mathbf{k}_{\mathrm{cat}} / K_{\mathrm{M}}\left(\mathrm{min}^{-1} \mu \mathrm{M}^{-1}\right)$ \\
\hline$[\mathrm{Ub}]_{2}{ }^{-6,48} \mathrm{Ub}$ & $15.5 \pm 1.5$ & $1.25 \pm 0.04$ & $0.080 \pm 0.008$ \\
{$[\mathrm{Ub}]_{2}{ }^{-11,48} \mathrm{Ub}$} & $52.2 \pm 8.8$ & $3.21 \pm 0.27$ & $0.062 \pm 0.012$ \\
{$[\mathrm{Ub}]_{2}{ }^{48,63} \mathrm{Ub}$} & $74.6 \pm 13.6$ & $0.38 \pm 0.03$ & $0.005 \pm 0.001$ \\
\hline
\end{tabular}

Figure 2. UCH37-RPN13 ${ }^{\mathrm{C}}$ preferentially binds and deubiquitinates $\mathrm{K} 6 / \mathrm{K} 48$ branched $\mathrm{Ub}_{3}$. (A) Binding affinities between His-TEV-UCH37(C88S)-RPN13C and various polyUb chains were measured by microscale thermophoresis. Binding isotherms (Figure S2A) were fit with a single-site binding model; best-fit $K_{\mathrm{D}}$ values are shown with standard errors. (B) Schematic of the free Ub sensor-based deubiquitination assay. (C) Michaelis-Menten kinetics of branched $\mathrm{Ub}_{3}$ hydrolysis by NS-UCH37-RPN13 ${ }^{\mathrm{C}}$. The table shows best-fit $K_{\mathrm{M}}$, $\mathrm{k}_{\mathrm{cat}}$, and $\mathrm{k}_{\mathrm{cat}} / K_{\mathrm{M}}$ values with standard errors from two independent replicates. 
bioRxiv preprint doi: https://doi.org/10.1101/2021.08.17.456719; this version posted August 17, 2021. The copyright holder for this preprint (which was not certified by peer review) is the author/funder. All rights reserved. No reuse allowed without permission.
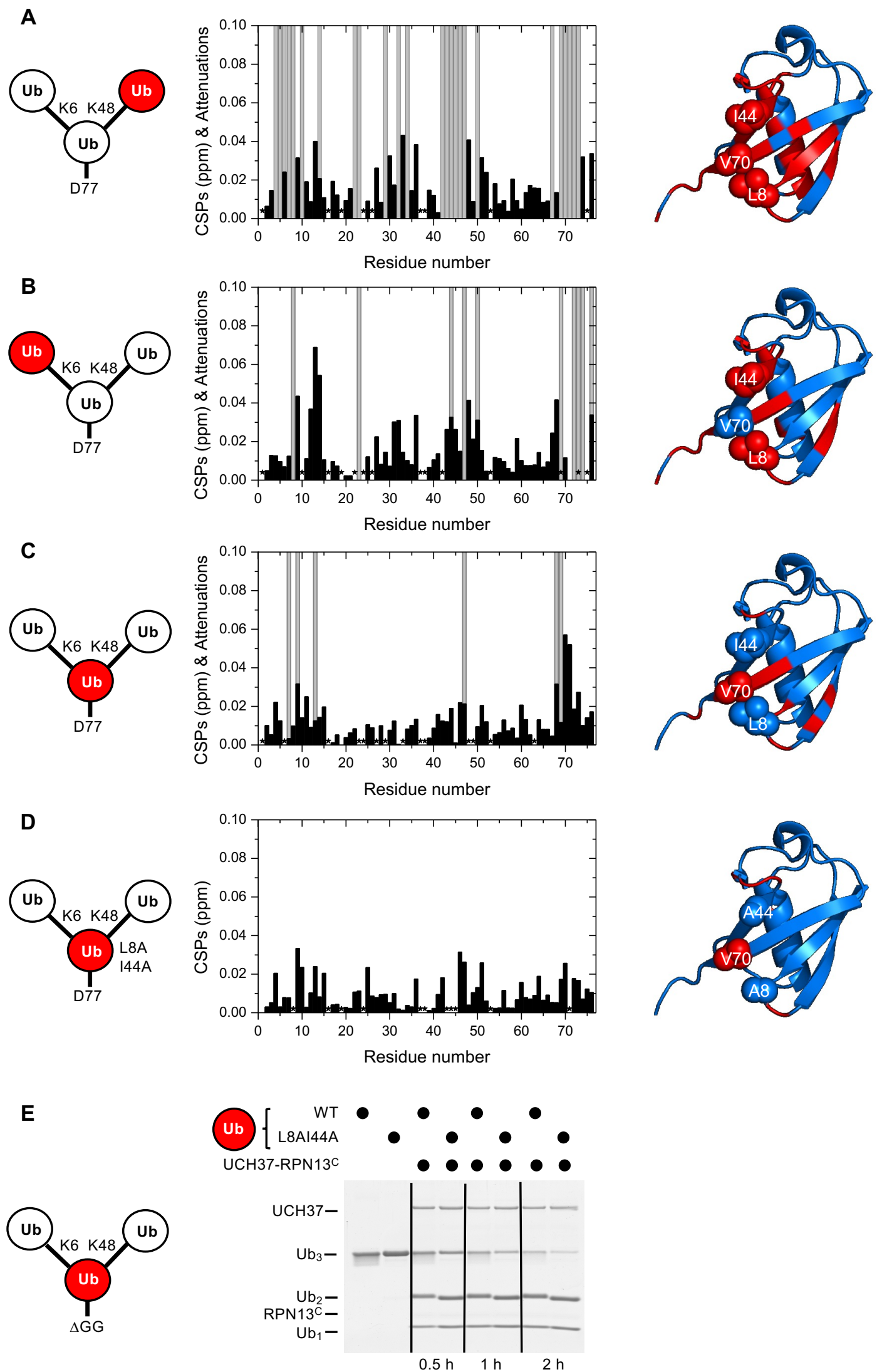

Figure 3. UCH37-RPN13 ${ }^{\mathrm{C}}$ contacts the hydrophobic patches on both distal Ub units in a branched $\mathrm{Ub}_{3}$ chain. (A-D) Residue-specific perturbations of backbone amide NMR signals in the (A) K48-linked distal Ub, (B) K6-linked distal $\mathrm{Ub},(\mathrm{C})$ the proximal $\mathrm{Ub}$, and $(\mathrm{D})$ mutated proximal $\mathrm{Ub}(\mathrm{L} 8 \mathrm{~A}, \mathrm{I44A})$ in branched $\mathrm{K} 6 / \mathrm{K} 48$ linked $\mathrm{Ub}_{3}$ caused by the addition of 1.2 molar equivalents of co-purified UCH37(C88A)-RPN13C. The NMR spectra are shown in Figure S3. Black bars represent chemical shift perturbations (CSPs, in ppm), grey bars mark residues exhibiting strong signal attenuations. Residues that were not observed or could not be unambiguously assigned/quantified due to signal overlap are marked with asterisks. Residues with strong signal attenuations or CSP $>0.025 \mathrm{ppm}$ are mapped (red) on the 3-D structure of Ub (PDB code: 1UBQ); the hydrophobic patch residues are shown in sphere representation. (E) $1 \mu \mathrm{M}$ UCH37-RPN13 ${ }^{\mathrm{C}}$ were incubated with $5 \mu \mathrm{M}$ substrate as indicated at $37^{\circ} \mathrm{C}$. Reaction products were analyzed by SDSPAGE and Coomassie staining. 
A

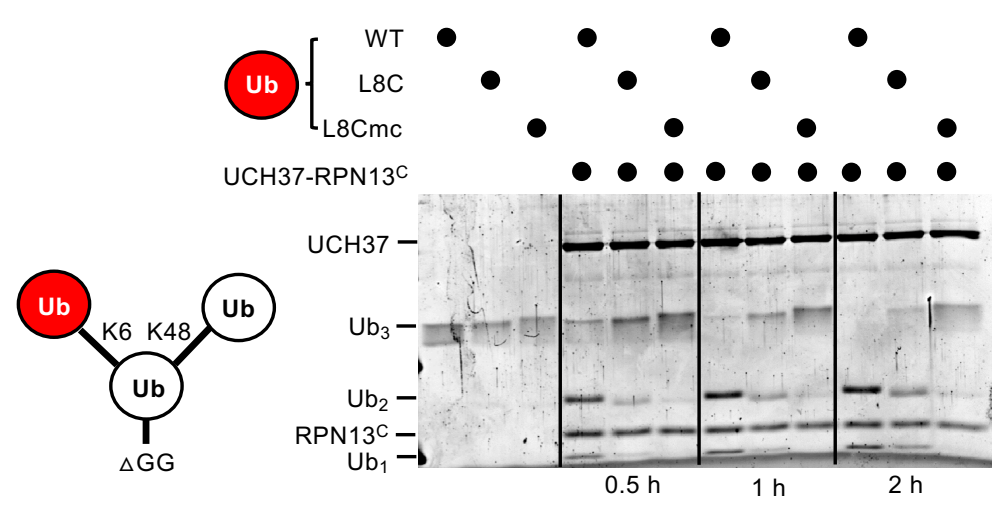

B

Native
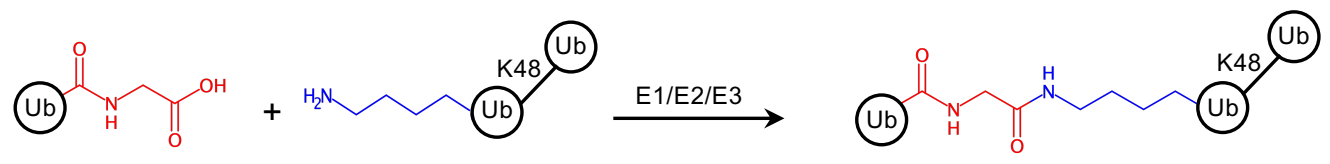

Mimic1 His-(ub)

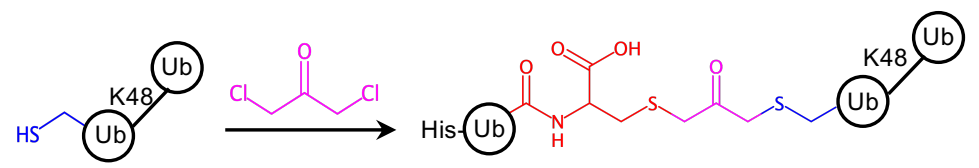

Mimic2 His-(Ub)

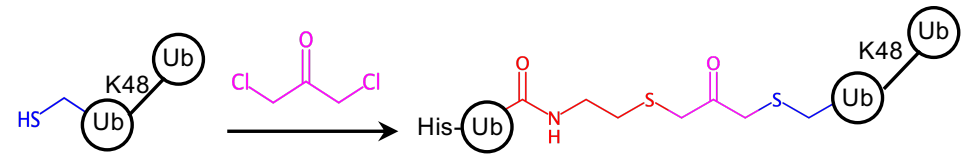

C
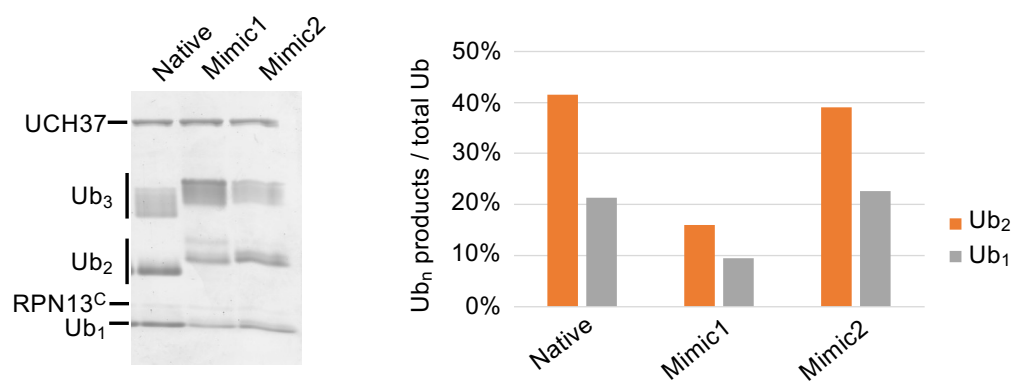

D

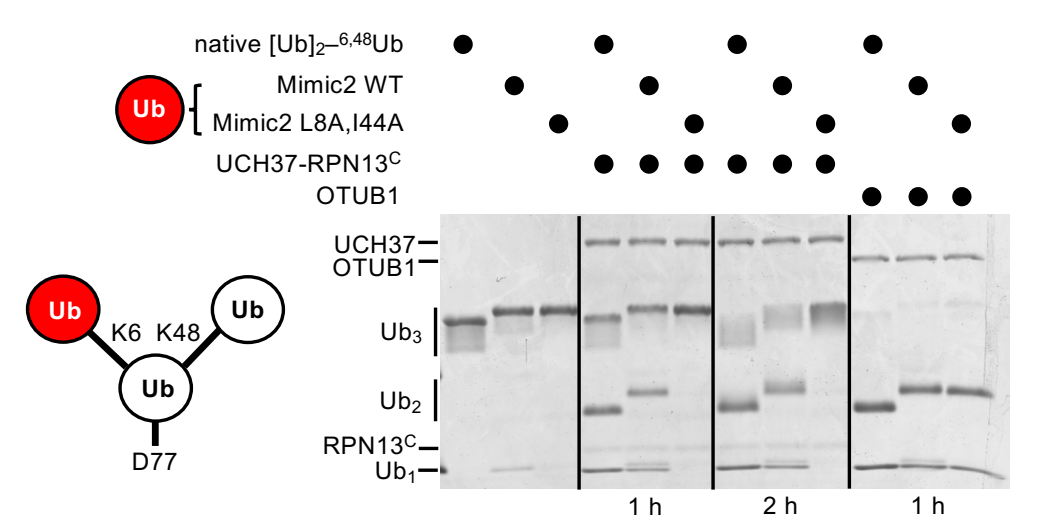

Figure 4. The hydrophobic patch on the K6-linked distal $\mathrm{Ub}$ is required for debranching. (A) $1 \mu \mathrm{M}$ Enzyme was incubated with $5 \mu \mathrm{M}$ of the indicated substrate at $37^{\circ} \mathrm{C}$; at the times indicated, aliquots were taken and analyzed by SDS-PAGE and Coomassie staining. (B) Schematic showing assembly strategies and structures of branched K6/K48$\mathrm{Ub}_{3}$ and crosslinked mimics. (C) Comparison of native K6/K48-Ub 3 with Mimic 1 and 2 in gel-based DUB assays using NS-UCH37-RPN13C as described in (A). Quantification of $\mathrm{Ub}_{2}$ and $\mathrm{Ub}_{1}$ products from the gel are plotted as shown. (D) Native K6/K48-Ub $b_{3}$ or Mimic2 $\mathrm{Ub}_{3}$ containing either wild-type or L8A,I44A mutant $\mathrm{Ub}$ crosslinked to $\mathrm{Ub}-48 \mathrm{Ub}(\mathrm{K} 6 \mathrm{C})$ were analyzed by gel-based DUB assays as described in (A). 
A

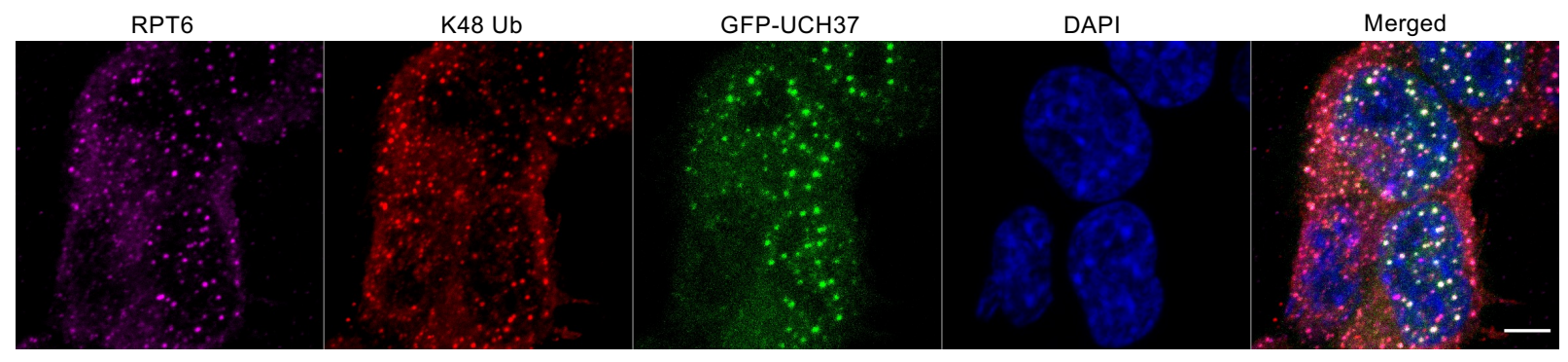

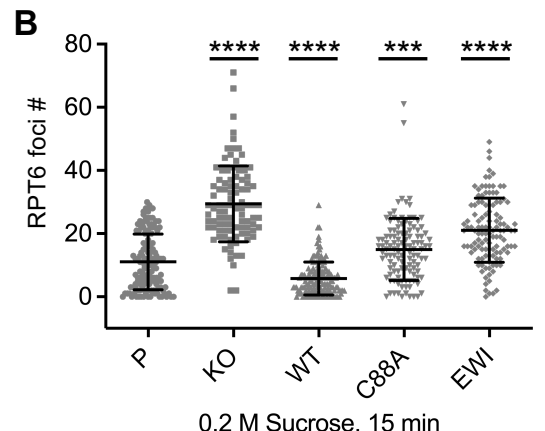

$0.2 \mathrm{M}$ Sucrose, $15 \mathrm{~min}$

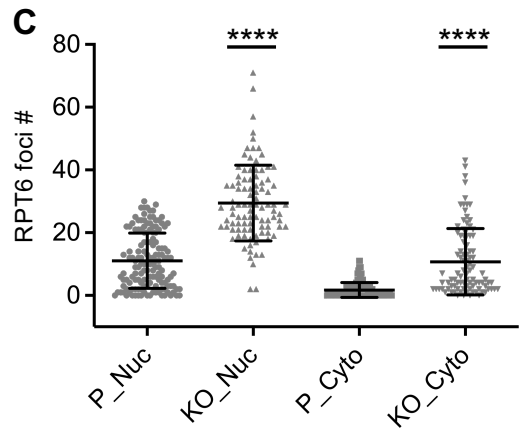

$0.2 \mathrm{M}$ Sucrose, $15 \mathrm{~min}$

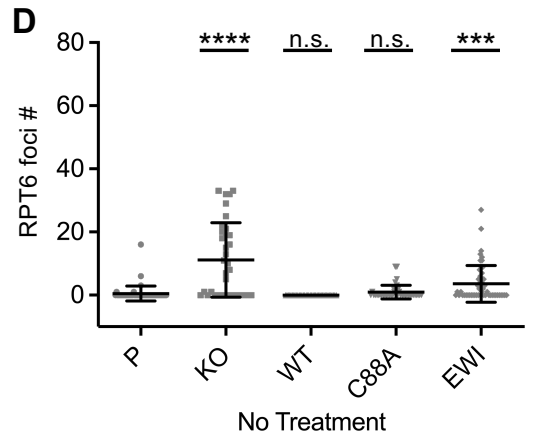

$\mathbf{F}$

$\mathbf{F}$
E

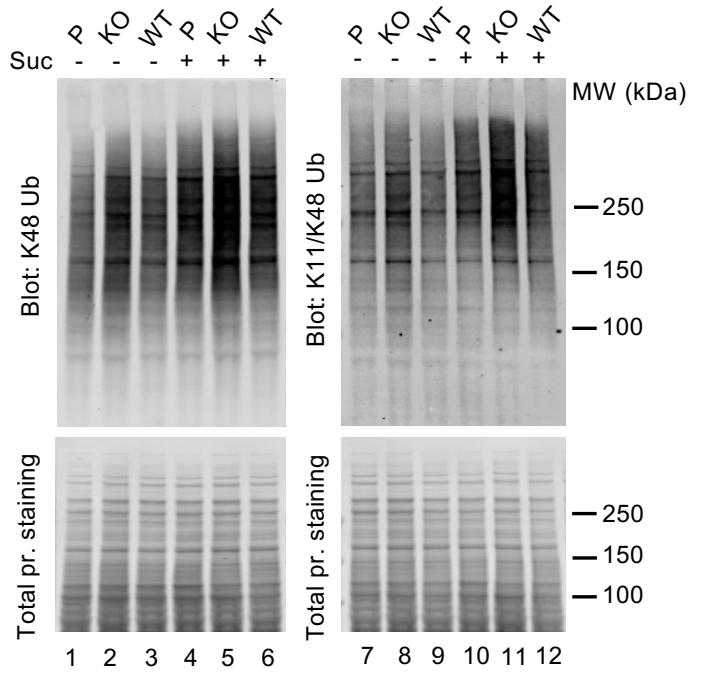

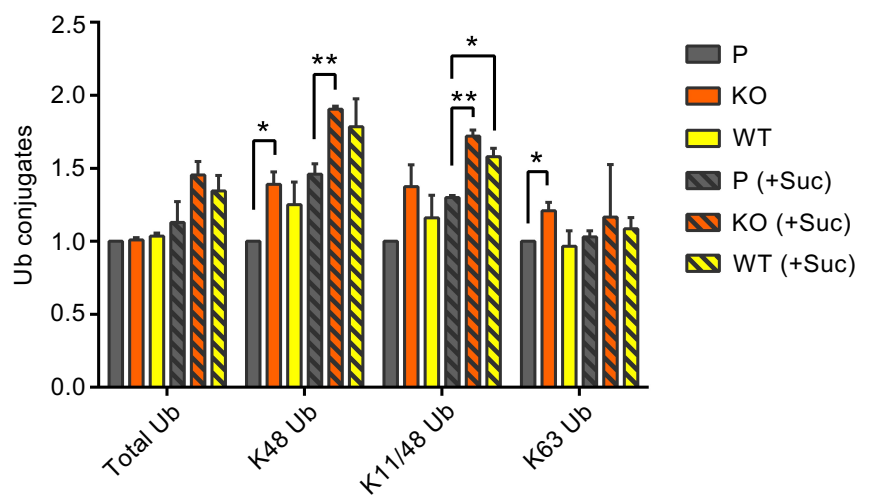

Figure 5. UCH37 activity regulates proteasome condensates upon osmotic stress. (A) HCT116 cells expressing Flag-GFP-UCH37(C88A) were treated with $0.2 \mathrm{M}$ sucrose for $15 \mathrm{~min}$, then fixed and immunostained with RPT6 and K48-specific antibodies. (B-D) RPT6 foci numbers in each cell were quantified and are shown as mean \pm SD; $n>100$ cells were measured for each cell type. Unpaired t-tests were performed between each cell type and $\mathrm{P}:{ }^{* * *}, p<0.001$; ${ }^{* * * *}, p<0.0001$; n.s., not significant. (E) Whole-cell lysates were collected from $\mathrm{P}, \mathrm{KO}$ and WT cells with or without 30 min $0.2 \mathrm{M}$ sucrose treatment, and then analyzed by SDS-PAGE and immunblotting with the indicated antibodies. (F) Quantification of Ub conjugates from whole-cell lysates immunoblotted with FK2 antibody (for total Ub conjugates) or with linkage-specific anti-Ub antibodies. Representative blots are shown in (E). Anti-Ub signals relative to those in $P$ were plotted after normalization using the signal intensities from total-protein stain. Mean \pm SD from two independent replicates are plotted. Unpaired t-tests were performed between each cell type and $\mathrm{P}:{ }^{*}, p<0.05 ;{ }^{* *}, p<0.01$. 
A

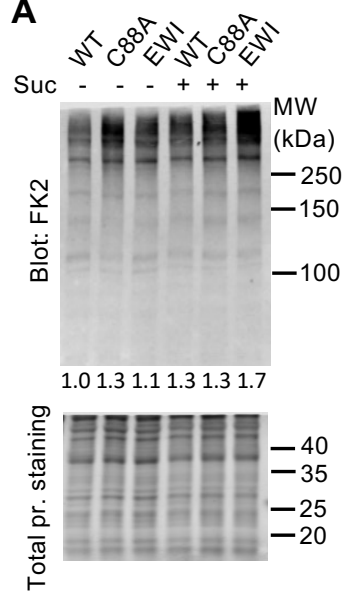

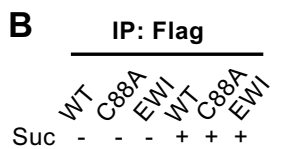

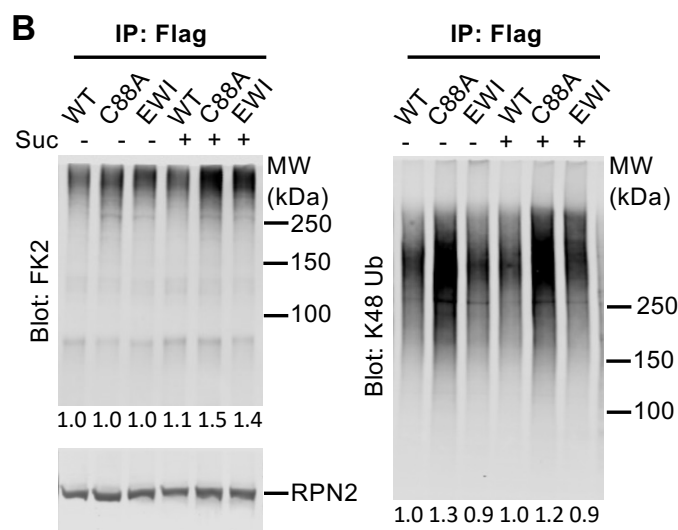

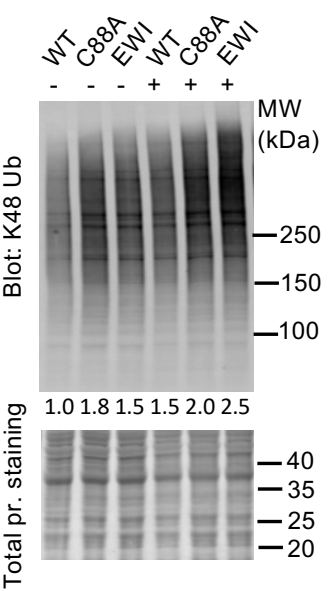
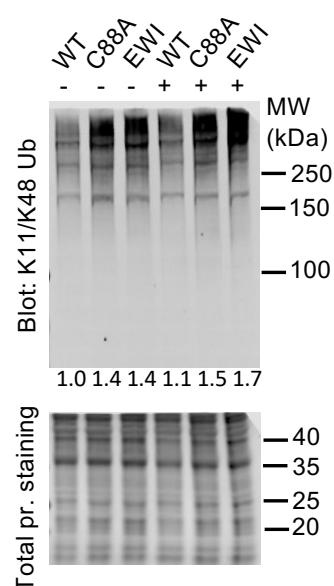

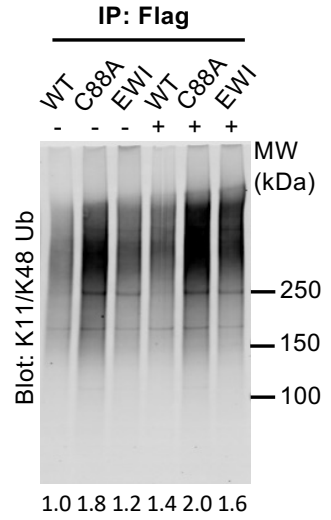

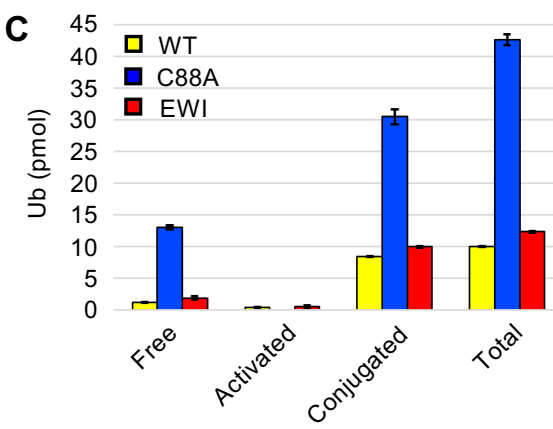

D

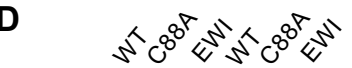

Suc
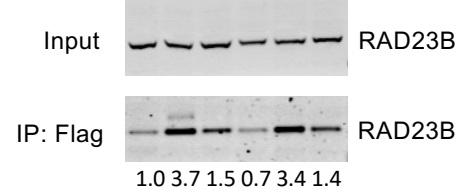

E

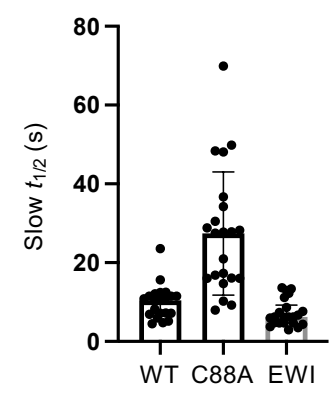

$\mathbf{F}$

PIPs

$\square$ WT $\square$ C88S $\square$ EWI

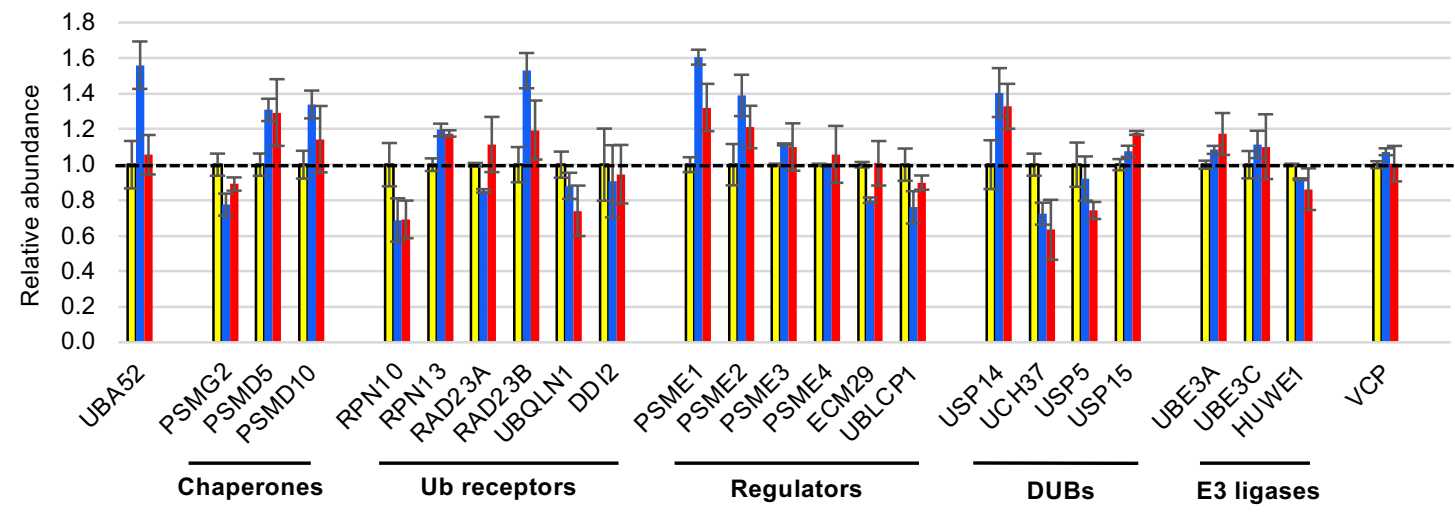

Figure 6. UCH37(C88A)-containing proteasomes accumulate polyUb species and RAD23B. (A) Soluble cell lysates were collected from WT, C88A and EWI cells with or without $30 \mathrm{~min} 0.2 \mathrm{M}$ sucrose treatment, analyzed by SDS-PAGE and immunblotting with indicated antibodies. Numbers indicate Ub signals relative to those in untreated WT cells after normalization against total protein stain signals. (B) UCH37-containing proteasomes were

immunoprecipitated and analyzed as described in (A). Numbers below the lanes indicate $\mathrm{Ub}$ signals relative to those in untreated WT cells after normalization against RPN2 signals. (C) Different types of Ub species associated with UCH37containing proteasomes immunoprecipitated from HEK293 cells were quantified by a free Ub sensor-based assay ${ }^{31}$.

Shown are mean \pm SD from two independent replicates. (D) RAD23B accumulation detected on UCH37(C88A)containing proteasomes as described in (A) and (B). (E) FRAP analysis of GFP-UCH37 after $0.2 \mathrm{M}$ sucrose treatment demonstrates that C88A-containing proteasomes are less mobile. At least 20 foci from 7 or 8 cells were analyzed from each cell line. After fitting the FRAP curve with two exponentials, the slow $t_{1 / 2}$ of each focus were plotted with mean and SD indicated. (F) UCH37-containing proteasomes from WT, C88S and EWI cells were isolated with immobilized antiGFP nanobodies, trypsin digested, and the peptides analyzed by TMT mass spectrometry. Protein abundances were measured from two independent pulldowns, each analyzed by mass spectrometry in triplicate, and plotted as mean \pm SD after normalizing against signals from WT samples. 


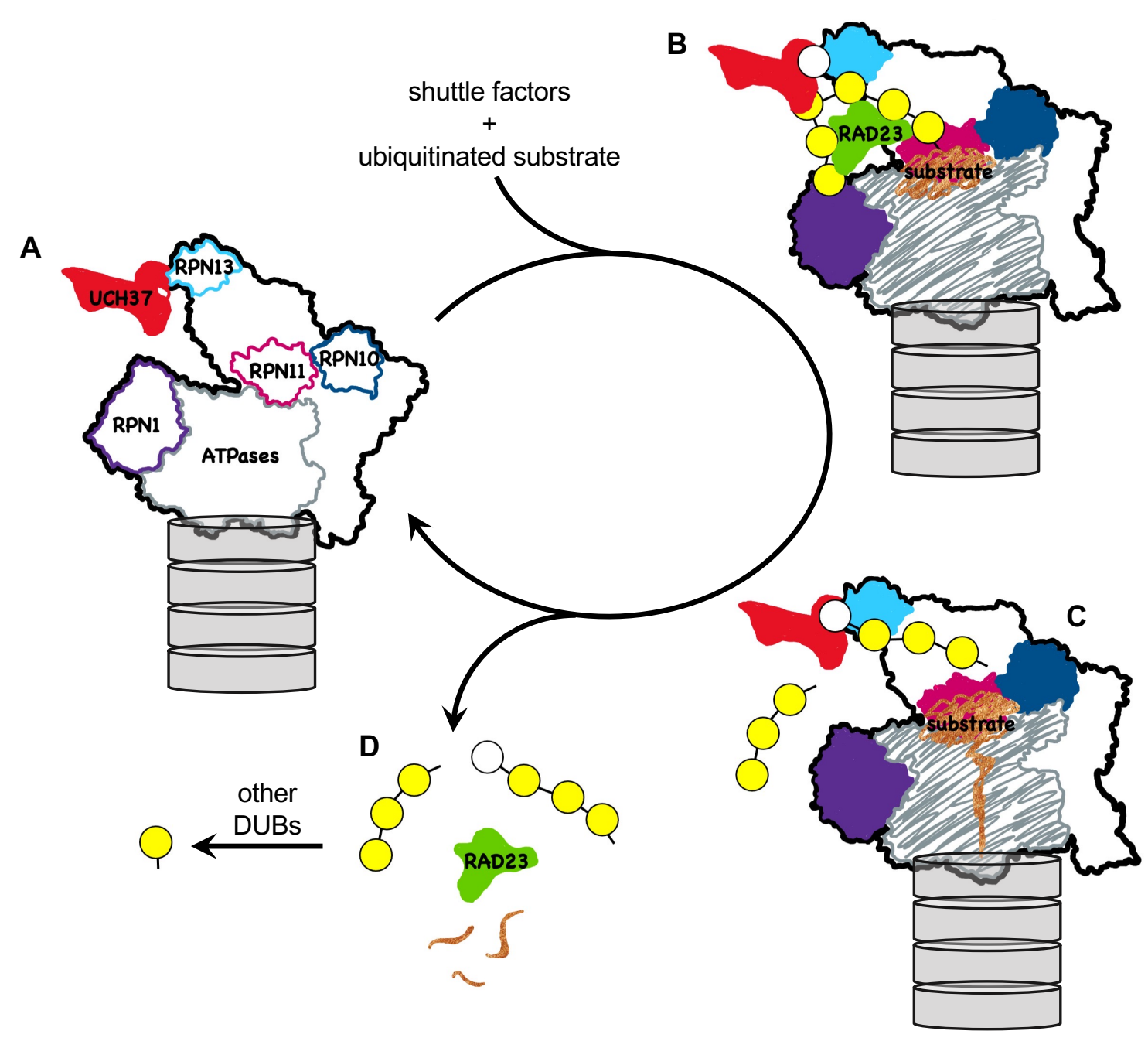

Figure 7. A model for how UCH37 promotes substrate processing and recycling of the proteasome through debranching of polyUb chains. (A) A schematic of singly-capped 26S proteasome in s1 state (PDB code: 4CR2) with locations indicated for intrinsic Ub receptors (RPN1, RPN10, RPN13) and associated DUBs (RPN11, UCH37); for clarity, USP14 is not shown. (B) RAD23 delivers a substrate (brown) modified by polyUb with a single branch point. $\mathrm{UCH} 37$ binds both distal Ubs at the branch point. K48-linked Ubs are shown in yellow whereas a non-K48-linked Ub is shown in white. (C) A substrate-engaged proteasome (PDB code: 4CR4) where substrate starts to be translocated and polyUb is removed en bloc by RPN11. Whether debranching by UCH37 occurs before or after RPN11 action is unknown. (D) UCH37 cleavage of the K48-linkage at the branch point facilitates release of free (poly)Ub chains and the shuttle factors. 
A Nomenclature for homotypic and heterotypic Ub chains (in the order of distal - proximal)

$\begin{array}{lll}\mathrm{K} 48 \mathrm{Ub}_{2} & = & \mathrm{Ub} \text { 48 }^{48 \mathrm{Ub}} \\ \mathrm{K} 6 \mathrm{Ub}_{3} & = & \mathrm{Ub}{ }^{6} \mathrm{Ub}-6 \mathrm{Ub} \\ \mathrm{K} 11 \mathrm{Ub}_{3} & = & \mathrm{Ub}{ }^{11} \mathrm{Ub}-11 \mathrm{Ub} \\ \mathrm{K} 48 \mathrm{Ub}_{3} & = & \mathrm{Ub}-48 \mathrm{Ub}-48 \mathrm{Ub} \\ \mathrm{K} 63 \mathrm{Ub}_{3} & = & \mathrm{Ub}{ }^{63} \mathrm{Ub}{ }^{63} \mathrm{Ub} \\ & & \\ \mathrm{K} 6 / \mathrm{K} 48 \mathrm{Ub}_{3} & = & {[\mathrm{Ub}]_{2}-^{6,48} \mathrm{Ub}} \\ \mathrm{K} 11 / \mathrm{K} 48 \mathrm{Ub}_{3} & = & {[\mathrm{Ub}]_{2}-11,48 \mathrm{Ub}} \\ \mathrm{K} 48 / \mathrm{K} 63 \mathrm{Ub}_{3} & = & {[\mathrm{Ub}]_{2}-48,63 \mathrm{Ub}} \\ \mathrm{K} 6-\mathrm{K} 48 \mathrm{Ub}_{3} & = & \mathrm{Ub}-6 \mathrm{Ub}-48 \mathrm{Ub} \\ \mathrm{K} 48-\mathrm{K} 6 \mathrm{Ub}_{3} & = & \mathrm{Ub}-48 \mathrm{Ub}-6 \mathrm{Ub}\end{array}$

B

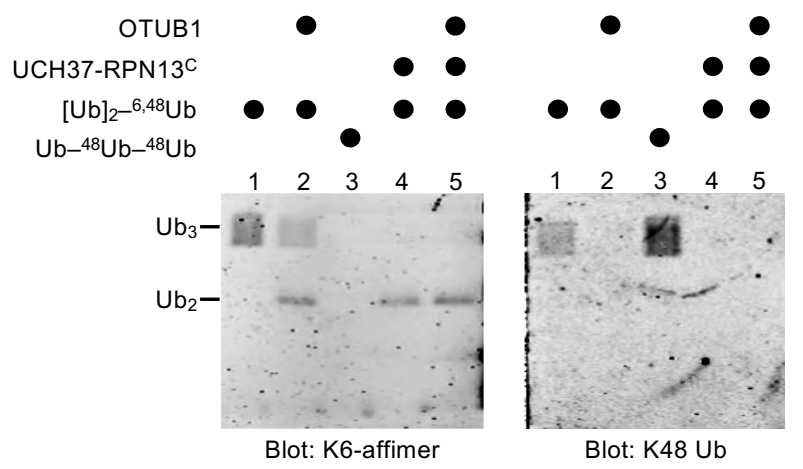

C

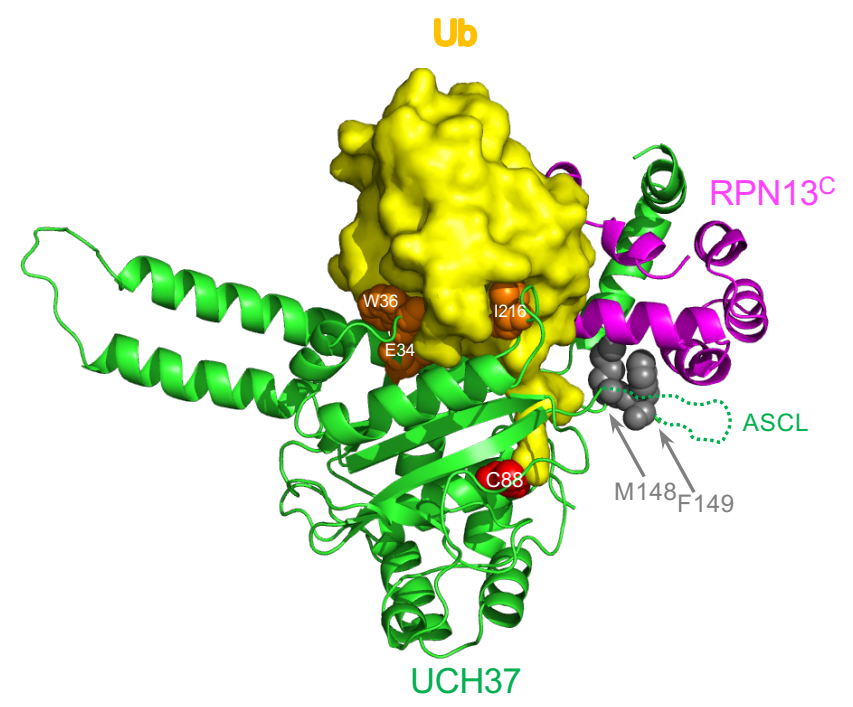

Supplemental Figure S1. (A) Notation used to describe architectures of polyUb chains used in this paper (adopted from $^{48}$ ). (B) $\cup C H 37$ exclusively cleaves the $\mathrm{K} 48$ linkage in a branched chain. Ub 3 substrates were incubated with the indicated DUB (i.e., UCH37-RPN13 ${ }^{\mathrm{C}}$ complex or OTUB1) and the reaction products were detected using either the K6 linkage-specific anti-Ub affimer ${ }^{45}$ or K48 linkage-specific anti-Ub antibody. In Lane 5, OTUB1 was added after UCH37RPN13 ${ }^{C}$ had completely hydrolyzed the $\mathrm{K} 6 / \mathrm{K} 48$ branched $\mathrm{Ub}_{3}$, thereby producing $\mathrm{K} 6$-linked $\mathrm{Ub}_{2}$ that was refractory to OTUB1. (C) Crystal structure of the UCH37-RPN13C_Ub complex (PDB code: 4WLR). Part of the ASCL is disordered and is represented as a dotted line. UCH37 ASCL residues M148 F149 contact RPN13C and are shown in gray. $\mathrm{UCH} 37$ residues E34, W36 and $\mathrm{I} 216$ contact the hydrophobic patch on $\mathrm{Ub}$ and are shown in orange. UCH37 catalyticsite residue $\mathrm{C} 88$ is shown in red. 
A

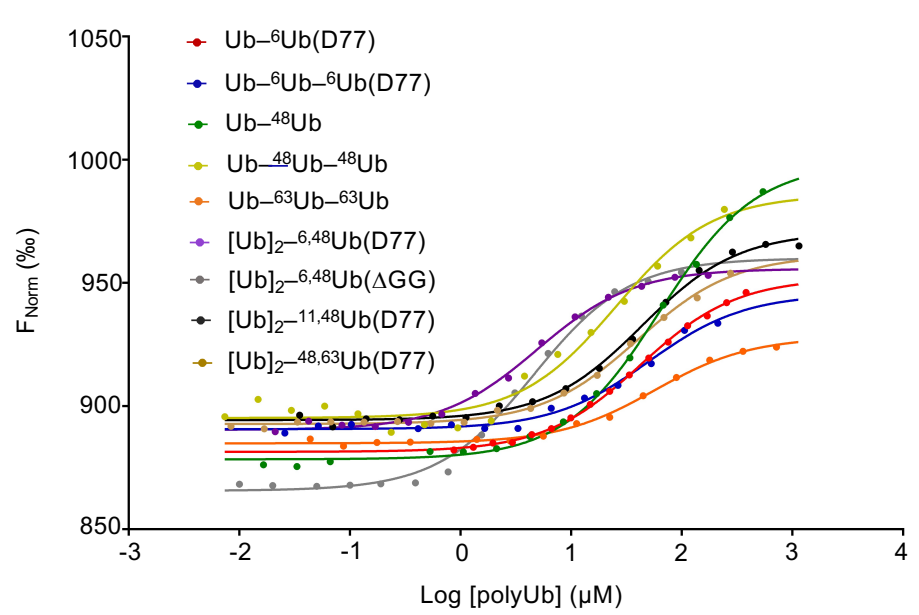

B

\begin{tabular}{|c|c|c|c|}
\hline UCH37 preparation & Description & $\begin{array}{l}\text { Specific Activity for } \\
\text { Ub-AMC } \\
\left(A U \cdot \min ^{-1} \cdot \mathrm{nM}^{-1}\right)\end{array}$ & $\begin{array}{l}\text { Relative } \\
\text { Activity }\end{array}$ \\
\hline His-TEV-UCH37-RPN13 ${ }^{\mathrm{C}}$ & $\begin{array}{l}\text { His-TEV-tagged } \mathrm{UCH} 37 \text { was preincubated with RPN } 13^{\mathrm{C}} \text { right } \\
\text { before enzymatic assays }\end{array}$ & 9.01 & $100 \%$ \\
\hline NS-UCH37-RPN13 ${ }^{\mathrm{C}}$ & $\begin{array}{l}\text { His tag was removed by } T E V \text { protease, leaving an N-terminal } \\
\text { serine that replaces Met } 1 \text { in UCH37. NS-UCH37 was } \\
\text { preincubated with RPN1 } 1{ }^{C} \text { right before enzymatic assays. }\end{array}$ & 12.58 & $140 \%$ \\
\hline co-purified NS-UCH37-RPN13 & $\begin{array}{l}\text { His-TEV-UCH } 37 \text { were co-purified with GST-TEV-RPN } 13^{\mathrm{C}} \text { and } \\
\text { the tags were removed by TEV protease. }\end{array}$ & 14.97 & $166 \%$ \\
\hline co-purified NS-UCH37-RPN13 ${ }^{\mathrm{FL}}$ & $\begin{array}{l}\text { His-TEV-UCH37 were co-purified with full-length His-RPN13 } \\
\text { and the N-terminal tag on UCH37 was removed by TEV } \\
\text { protease. }\end{array}$ & 7.10 & $79 \%$ \\
\hline
\end{tabular}

C

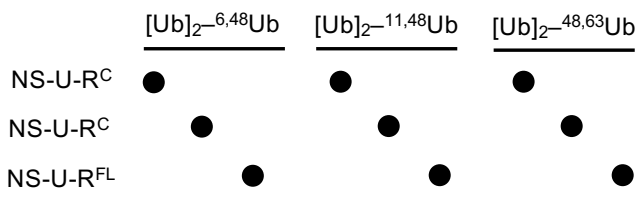

co-purified NS-U-R ${ }^{\mathrm{FL}}$
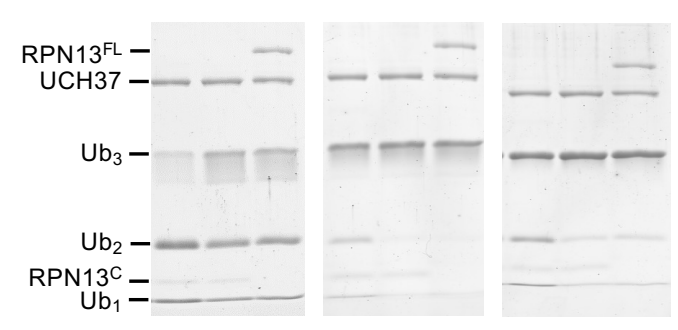

\section{D}

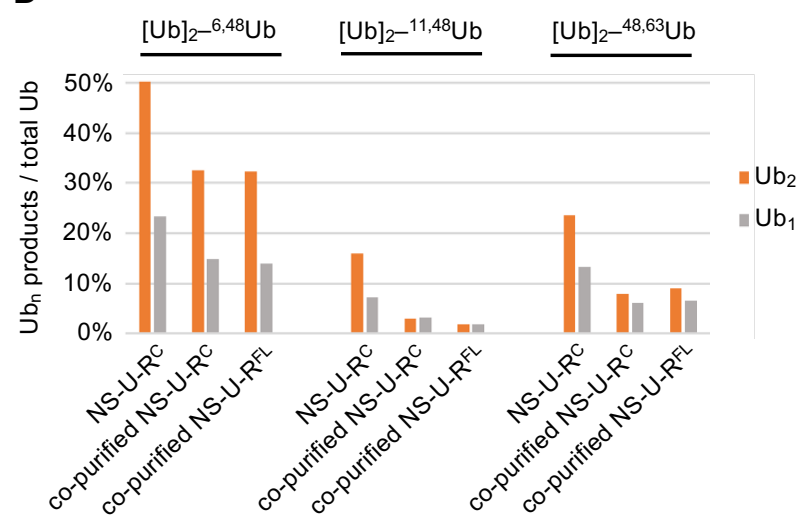

Supplemental Figure S2. (A) Ub chain binding isotherms of His-TEV-UCH37(C88S)-RPN13C were determined by microscale thermophoresis; fluorescent labeling used RED-tris-NTA that binds to the His-tag. Lines show the fitted curves using a single-site binding model. (B) Each UCH37 preparation was evaluated by Ub-AMC hydrolysis. Specific activities were determined with $1 \mathrm{nM}$ enzyme and $8 \mu \mathrm{M}$ Ub-AMC; AU, arbitrary fluorescence unit. (C) Gel-based DUB assays were performed to compare debranching efficiencies by the $\mathrm{UCH} 37$ preparations described in (B). Enzymes (1 $\mu \mathrm{M})$ were incubated with $5 \mu \mathrm{M}$ substrate as indicated for $2 \mathrm{~h}$ at $37^{\circ} \mathrm{C}$. Reaction products were quantified and plotted in (D). U-R ${ }^{\mathrm{C}}$ and $\mathrm{U}-\mathrm{R}^{\mathrm{FL}}$ represent $\mathrm{UCH} 37-\mathrm{RPN}^{1} 3^{\mathrm{C}}$ and $\mathrm{UCH} 37-\mathrm{RPN} 13^{\mathrm{FL}}$, respectively. 

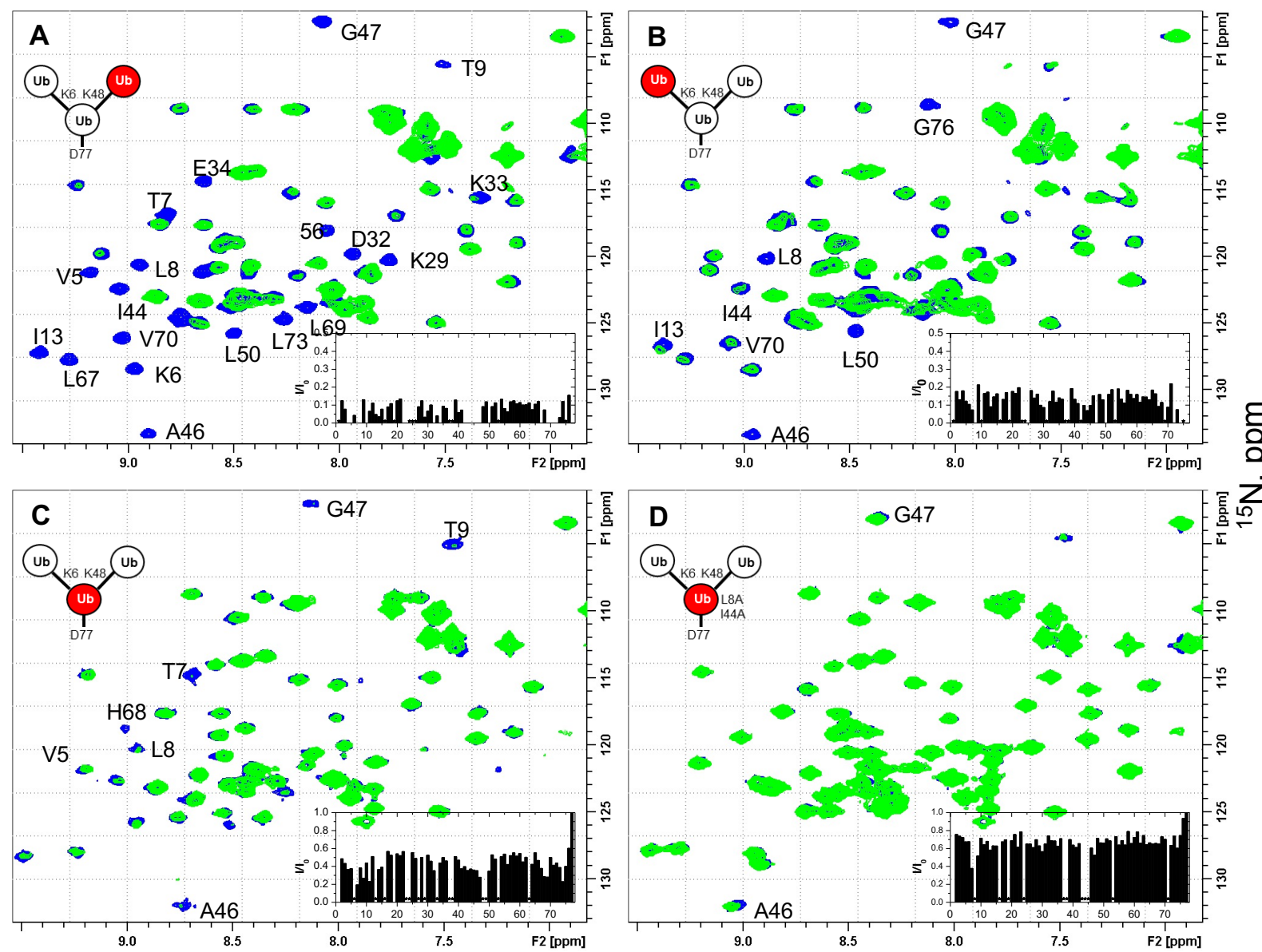

${ }^{1} \mathrm{H}, \mathrm{ppm}$

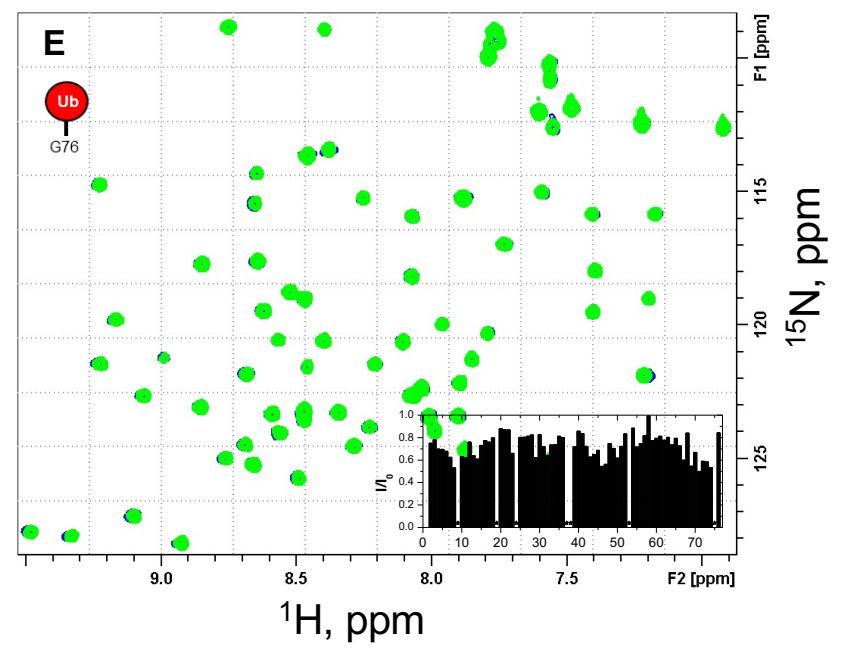

Supplemental Figure S3. NMR spectra show unit-specific $\mathrm{Ub}_{3}$ binding to UCH37-RPN13C. Overlays of ${ }^{1} \mathrm{H}-{ }^{15} \mathrm{~N}$ Overlays of ${ }^{1} \mathrm{H}-{ }^{15} \mathrm{~N}$ SOFAST-HMQC spectra $\left(\right.$ at $23^{\circ} \mathrm{C}$ ) of the individual ${ }^{15} \mathrm{~N}$-labeled Ub units in the branched $\mathrm{K} 6 / \mathrm{K} 48$ linked $\mathrm{Ub}_{3}$ and of monomeric $\mathrm{Ub}$ before (blue) and after (green) the addition of a 1.2 molar equivalent of $\mathrm{UCH} 37(\mathrm{C} 88 \mathrm{~S})-\mathrm{RPN13}$. Shown are fragments of the 'fingerprint' region of the spectra of (A) K48-linked distal Ub, (B) K6-linked distal Ub, (C) proximal Ub D77, and (D) proximal Ub D77 (L8A, 144A), as well as (E) monomeric WT Ub. Residues exhibiting significant signal attenuations as well as hydrophobic patch residues are indicated. The insets depict the ratio $\left(I / I_{0}\right)$ of signal peak intensities after $(I)$ and before $\left(I_{0}\right)$ the addition of $U \mathrm{CH} 37(\mathrm{C} 88 \mathrm{~S})-\mathrm{RPN} 13^{\mathrm{C}}$ as a function of residue number. The spectra of unbound protein were recorded with $64(A, B), 128(C, D)$, or 32 scans $(E)$, those of the complex were recorded with $2048(A, B), 512(C)$, and $256(D, E)$ scans to compensate for the overall signal decrease as a result of binding. The overall attenuation of peak signal intensities $\left(I / I_{0}\right)$ caused by the addition of $\mathrm{UCH} 37$ (C88S)-RPN13 ${ }^{\mathrm{C}}$ was $0.09 \pm 0.03$ for K48-linked distal $\mathrm{Ub}$ and $0.14 \pm 0.04$ for K6-linked distal Ub (completely attenuated signals excluded), $0.43 \pm 0.09$ for the proximal $\mathrm{Ub}$ and $0.67 \pm 0.07$ for the proximal $\mathrm{Ub}$ with the L8A,I44A mutation (signals from flexible G76 and D77 excluded), and $0.73 \pm 0.10$ for monoUb. Residues that exhibited complete signal attenuation and those with $I / I_{0}<$ mean $-2 x$ S.D. (in A, B) or $I / I_{0}<$ mean $-1.5 x$ S.D. (in C) are marked with grey bars in Figure 3A-C. 


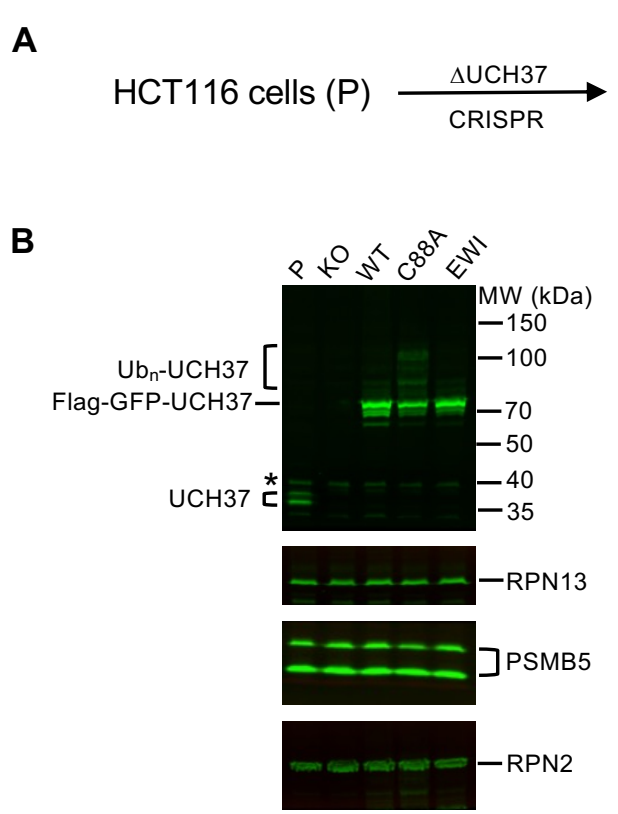

C
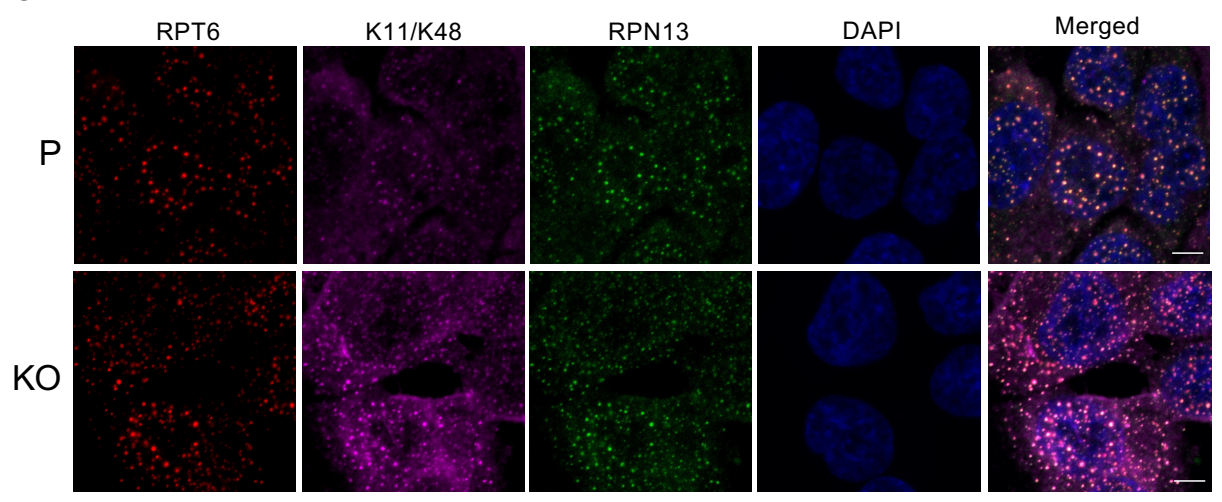

D

RPT6

K11/K48

DAPI

Merged
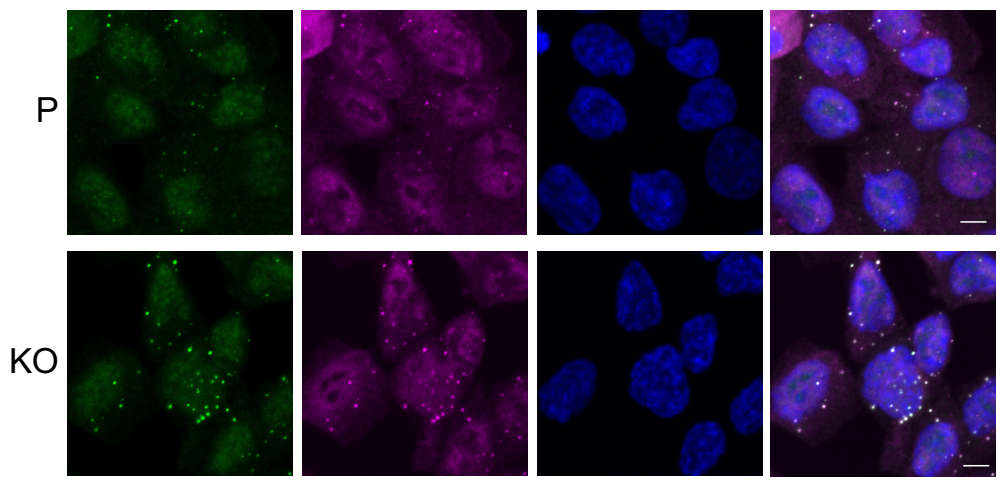

Supplemental Figure S5 (A) Schematic of cell line constructions. UCH37 knockout (KO) was derived from parental HCT116 cells $(P)$ by CRISPR. KO cells were then infected with retrovirus encoding wild-type, C88A, or EWI versions of Flag-GFP-UCH37, followed by stable cell line selection to create WT, C88A and EWI polyclonal cell lines. (B) Wholecell lysates from cells as described in (A) were analyzed by SDS-PAGE and immunoblotting; * denotes a non-specific band. Note that endogenous $\mathrm{UCH} 37$ has two isoforms, and $\mathrm{UCH} 37(\mathrm{C} 88 \mathrm{~A})$ is oligo-ubiquitinated. (C and D) Cells are treated with $0.2 \mathrm{M}$ sucrose for $15 \mathrm{~min}(\mathrm{C})$ or $0.5 \mathrm{mM}$ sodium arsenite for $90 \mathrm{~min}(\mathrm{D})$, fixed and immunostained with the indicated antibodies. 
A

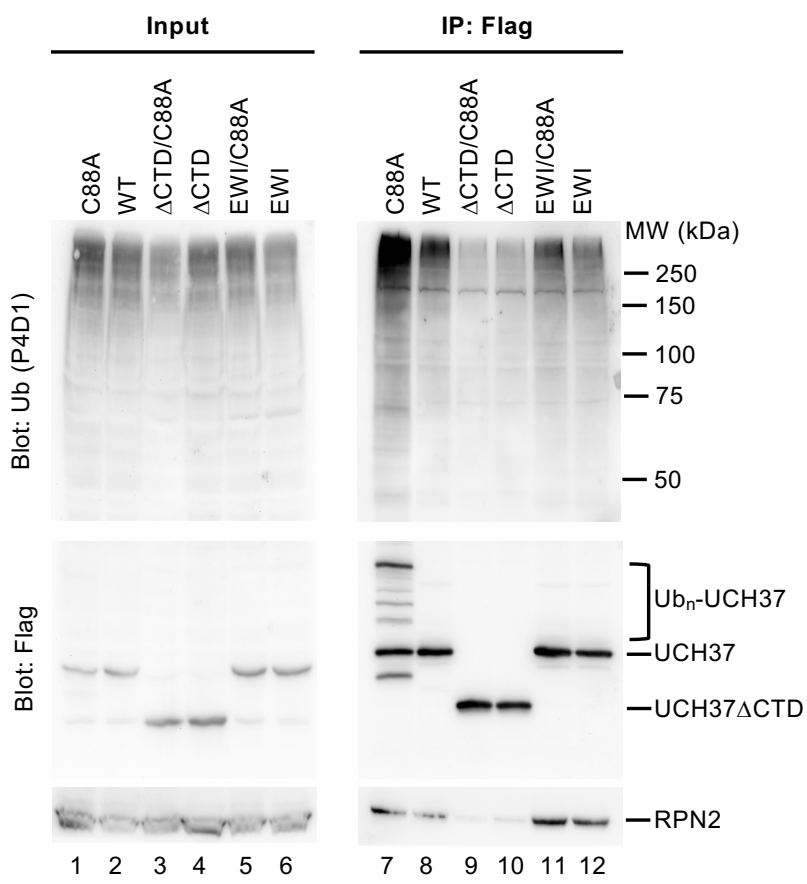

B
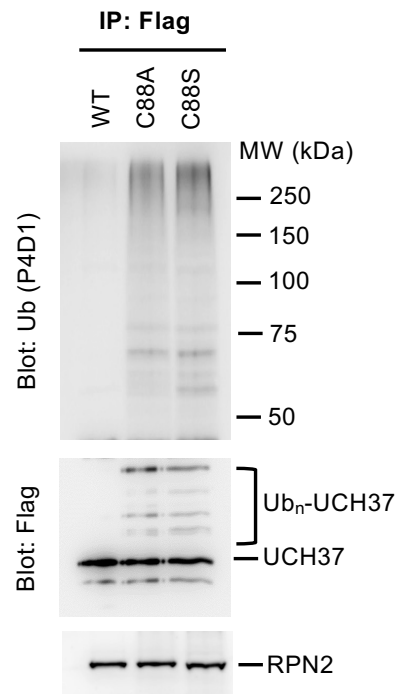

C

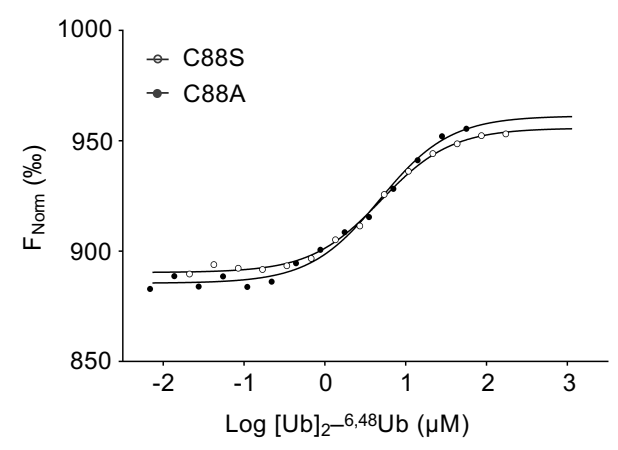

D

\begin{tabular}{|c|c|c|c|}
\hline & Ub chains & $\begin{array}{l}\text { Proximal Ub } \\
\text { modification }\end{array}$ & $K_{\mathrm{D}}(\mu \mathrm{M})$ \\
\hline \multirow[t]{3}{*}{ Linear } & $U b-6 \mathrm{Ub}--^{6} \mathrm{Ub}$ & D77 & $75.7 \pm 9.7$ \\
\hline & $\mathrm{Ub}-48 \mathrm{Ub}-48 \mathrm{Ub}$ & D77 & $1.6 \pm 0.6$ \\
\hline & $\mathrm{Ub}-63 \mathrm{Ub}-63 \mathrm{Ub}$ & - & $25.5 \pm 8.3$ \\
\hline \multirow[t]{3}{*}{ Branched } & {$[\mathrm{Ub}]_{2}-^{6,48} \mathrm{Ub}$} & $\Delta G G$ & $1.0 \pm 0.9$ \\
\hline & {$[\mathrm{Ub}]_{2^{-11,48} \mathrm{Ub}}$} & D77 & $1.6 \pm 0.5$ \\
\hline & {$[\mathrm{Ub}]_{2^{-}-48,63 \mathrm{Ub}}$} & D77 & $2.4 \pm 1.4$ \\
\hline
\end{tabular}

E

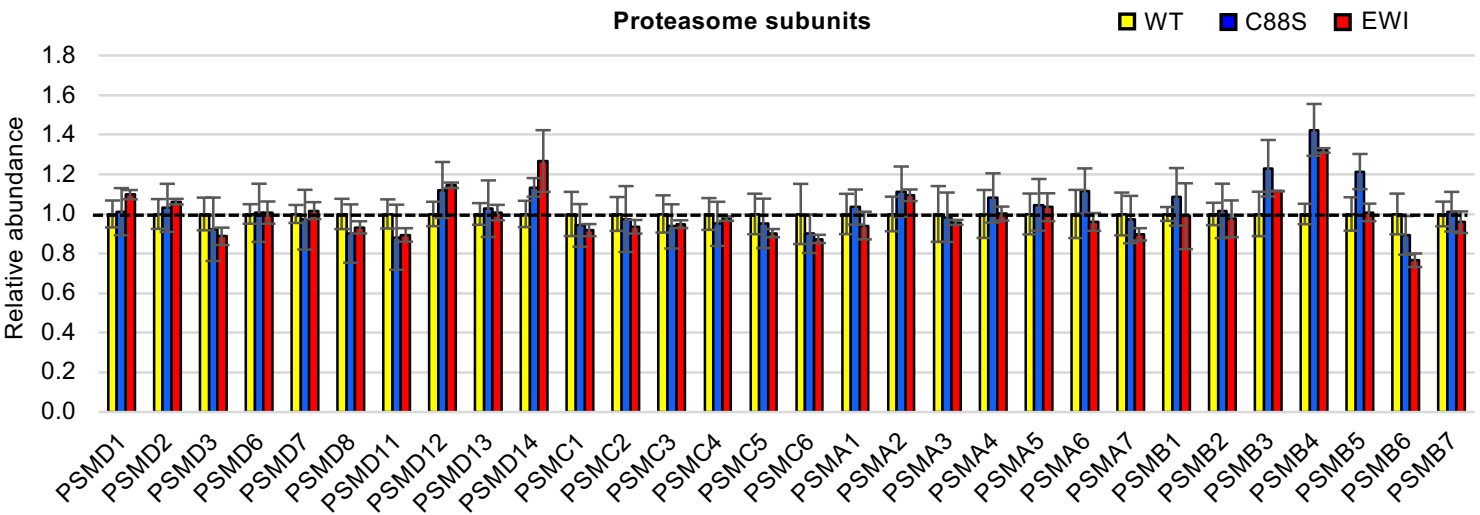

Supplemental Figure S6. (A) Analysis of UCH37-containing proteasomes from HEK293 cells. Flp-In T-REx 293 cell lines were constructed to inducibly express different versions of Flag-tagged UCH37. After Dox induction for $48 \mathrm{~h}$, antiFlag immunoprecipitates from the cells were analyzed by SDS-PAGE and immunoblotting with the indicated antibodies. (B) Anti-Flag immunoprecipitates from cells inducibly express UCH37 C88A or C88S were analyzed as described in (A). (C) Microscale thermophoresis were performed to determine binding affinities between UCH37(C88A), or $\mathrm{UCH} 37\left(\mathrm{C} 88 \mathrm{~S}\right.$ ), and $\mathrm{K} 6 / \mathrm{K} 48$ branched $\mathrm{Ub}_{3}$, as described in Figure 2A. (D) Microscale thermophoresis was performed to determine binding affinities between full-length RAD23B and $\mathrm{Ub}_{3}$ chains of different topologies. Shown are best-fit $K_{\mathrm{D}}$ values with standard errors from a single-site binding model. (E) TMT mass spectrometry analysis of UCH37containing proteasomes as described in Figure 6F. 
His-TEV-UCH 37
NS-UCH 37

UCH 37 delta

UCH37deltaCTD

His-TEV-UCH 37

NS-UCH 37

UCH37deltaCTD

His-TEV-UCH 37

NS-UCH 37

UCH37deltaCTD

His-TEV-UCH 37

NS-UCH 37

UCH37deltaCTD

His-TEV-UCH 37

NS-UCH 37

UCH37deltaCTD

His-TEV-UCH 37

NS-UCH 37

UCH37deltaCTD

His-TEV-UCH 37 TK 362

NS-UCH 37 TK 329

UCH37deltaCTD -- 237
MHHHHHHGKPIPNPLLGLDSTENLYFQGIDPFTMTGNAGEWCLMESDPGVFTELIKGFGC 60 -STGNAGEWCLMESDPGVFTELIKGFGC 27 -MTGNAGEWCLMESDPGVFTELIKGFGC 27

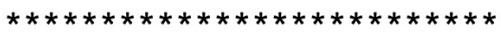

RGAQVEEIWSLEPENFEKLKPVHGLIFLFKWQPGEEPAGSVVQDSRLDTIFFAKQVINNA 120 RGAQVEEIWSLEPENFEKLKPVHGLIFLFKWQPGEEPAGSVVQDSRLDTIFFAKQVINNA 87 RGAQVEEIWSLEPENFEKLKPVHGLIFLFKWQPGEEPAGSVVQDSRLDTIFFAKQVINNA 87 $* * * * * * * * * * * * * * * * * * * * * * * * * * * * * * * * * * * * * * * * * * * * * * * * * * * * * * * * * * * *$

CATQAIVSVLLNCTHQDVHLGETLSEFKEFSQSFDAAMKGLALSNSDVIRQVHNSFARQQ 180 CATQAIVSVLLNCTHQDVHLGETLSEFKEFSQSFDAAMKGLALSNSDVIRQVHNSFARQQ 147 CATQAIVSVLLNCTHQDVHLGETLSEFKEFSQSFDAAMKGLALSNSDVIRQVHNSFARQQ 147 $* * * * * * * * * * * * * * * * * * * * * * * * * * * * * * * * * * * * * * * * * * * * * * * * * * * * * * * * * * * *$

MFEFDTKTSAKEEDAFHFVSYVPVNGRLYELDGLREGPIDLGACNQDDWISAVRPVIEKR 240 MFEFDTKTSAKEEDAFHFVSYVPVNGRLYELDGLREGPIDLGACNQDDWISAVRPVIEKR 207 MFEFDTKTSAKEEDAFHFVSYVPVNGRLYELDGLREGPIDLGACNQDDWISAVRPVIEKR 207 $* * * * * * * * * * * * * * * * * * * * * * * * * * * * * * * * * * * * * * * * * * * * * * * * * * * * * * * * * * * *$

IQKYSEGEIRFNLMAIVSDRKMIYEQKIAELQRQLAEEEPMDTDQGNSMLSAIQSEVAKN 300 IQKYSEGEIRFNLMAIVSDRKMIYEQKIAELQRQLAEEEPMDTDQGNSMLSAIQSEVAKN 267 IQKYSEGEIRFNLMAIVSDRKMIYEQKIAE----------------------------- 237 $* * * * * * * * * * * * * * * * * * * * * * * * * * * * * *$

QML IEEEVQKLKRYKIENIRRKHNYLPF IMELLKTLAEHQQLIPLVEKAKEKQNAKKAQE 360 QML IEEEVQKLKRYKIENIRRKHNYLPF IMELLKTLAEHQQLIPLVEKAKEKQNAKKAQE 327

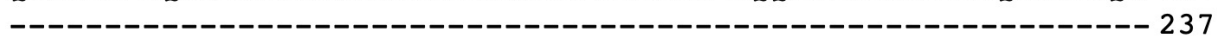

Supplemental Figure S7. Sequence alignment for UCH37 proteins used in this work. The active site C88 is highlighted in yellow and EWI residues are indicated in orange. 